\title{
Investigating ChaMPlane X\#Ray Sources in the Galactic Bulge with Magellan LDSS2 Spectra
}

\section{Citation}

Koenig, Xavier, Jonathan E. Grindlay, Maureen van den Berg, Silas Laycock, Ping Zhao, JaeSub Hong, and Eric M. Schlegel. 2008. "Investigating ChaMPlane X\#Ray Sources in the Galactic Bulge with Magellan LDSS2 Spectra." The Astrophysical Journal 685 (1): 463-77. https:// doi.org/10.1086/590206.

\section{Permanent link}

http://nrs.harvard.edu/urn-3:HUL.InstRepos:41399729

\section{Terms of Use}

This article was downloaded from Harvard University's DASH repository, and is made available under the terms and conditions applicable to Other Posted Material, as set forth at http:// nrs.harvard.edu/urn-3:HUL.InstRepos:dash.current.terms-of-use\#LAA

\section{Share Your Story}

The Harvard community has made this article openly available. Please share how this access benefits you. Submit a story.

Accessibility 


\title{
INVESTIGATING ChaMPlane X-RAY SOURCES IN THE GALACTIC BULGE WITH MAGELLAN LDSS2 SPECTRA
}

\author{
Xavier Koenig, ${ }^{1}$ Jonathan E. Grindlay, ${ }^{1}$ Maureen van den Berg, ${ }^{1}$ Silas Laycock, ${ }^{1}$ \\ Ping Zhao, ${ }^{1} \mathrm{~J}_{\mathrm{AeSub}}$ Hong, ${ }^{1}$ and Eric M. Schlegel ${ }^{2}$ \\ Received 2007 January 10; accepted 2008 May 15
}

\begin{abstract}
We have carried out optical and X-ray spectral analyses on a sample of 136 candidate optical counterparts of X-ray sources found in five Galactic bulge fields included in our Chandra Multiwavelength Plane Survey. We use a combination of optical spectral fitting and quantile X-ray analysis to obtain the hydrogen column density toward each object, and a three-dimensional dust model of the Galaxy to estimate the most probable distance in each case. We present the discovery of a population of stellar coronal emission sources, likely consisting of pre-main-sequence, young mainsequence, and main-sequence stars, as well as a component of active binaries of RS CVn or BY Dra type. We identify one candidate quiescent low-mass X-ray binary with a subgiant companion; we note that this object may also be an RS CVn system. We report the discovery of three new X-ray-detected cataclysmic variables (CVs) in the direction of the Galactic center (at distances $\lesssim 2 \mathrm{kpc}$ ). This number is in excess of predictions made with a simple CV model based on a local CV space density of $\lesssim 10^{-5} \mathrm{pc}^{-3}$, and a scale height $\sim 200 \mathrm{pc}$. We discuss several possible reasons for this observed excess.
\end{abstract}

Subject headings: Galaxy: stellar content — novae, cataclysmic variables — stars: activity — stars: late-type — surveys - X-rays: stars

Online material: color figure, machine-readable table

\section{INTRODUCTION}

The goal of the Chandra Multiwavelength Plane Survey (ChaMPlane) $^{3}$ is to study the Galactic X-ray point-source population, in particular accretion-powered X-ray sources. ChaMPlane (Grindlay et al. 2003, 2005) comprises two phases of study of the Galactic plane (Galactic latitudes $|b|<12^{\circ}$ ): first an X-ray survey of serendipitous sources from archival deep Chandra X-Ray Observatory pointings (with exposure times greater than $\sim 20 \mathrm{ks}$ ), and second an optical survey in $\mathrm{H} \alpha$ (narrowband) and Johnson $V, R$, and $I$ filters, using the Mosaic camera on the CTIO and KPNO $4 \mathrm{~m}$ telescopes to image $36^{\prime} \times 36^{\prime}$ fields centered on the Chandra pointings. Optical spectra are then obtained for classification of candidate optical counterparts to X-ray sources. Infrared (IR) observations are used to identify candidate counterparts in heavily obscured fields.

In this paper we analyze a sample of candidate optical counterparts from five ChaMPlane fields toward the Galactic bulge using low-resolution optical spectra. These fields are within $20^{\circ}$ of the Galactic center (GC), and within $3^{\circ}$ of the Galactic plane. Using the optical band necessarily constrains the scope of this project: stars at the distance of the GC $(\sim 8 \mathrm{kpc})$ are hidden behind a hydrogen column density $N_{\mathrm{H}} \sim(0.5-2.0) \times 10^{23} \mathrm{~cm}^{-2}$, and are thus absorbed by $A_{V} \gtrsim 25\left(A_{R} \gtrsim 19\right)$. Given our optical survey limit of $R=24$ and this level of extinction, optical counterparts at the $\mathrm{GC}$ are unobservable, and therefore our work is restricted to foreground $(d \lesssim 3 \mathrm{kpc})$ sources. As a consequence, we focus our efforts on two main goals: (1) to identify candidate cataclysmic variables (CVs) through their broad $\mathrm{H} \alpha$ line emission, and (2) to study the properties of the sample of stellar coronal emis-

\footnotetext{
1 Harvard-Smithsonian Center for Astrophysics, 60 Garden Street, Cambridge, MA 02138; xkoenig@cfa.harvard.edu.

2 Department of Physics and Astronomy, University of Texas at San Antonio, 1 UTSA Circle, San Antonio, TX 78249.

3 See http://hea-www.harvard.edu/ChaMPlane.
}

sion sources that we detect. In doing so we highlight anomalous cases as potential active binary or quiescent low-mass X-ray binary (qLMXB) systems.

The X-ray and optical data sets used in this study, and their reduction, are discussed in $\S \S 2$ and 3. In $\S 3$ we highlight new techniques developed for our analysis - a simple spectral fitting process to obtain the extinction $E(B-V)$ and thus $N_{\mathrm{H}}$ from the optical spectra, a three-dimensional (3D) dust model of the Galaxy (Drimmel \& Spergel 2001, hereafter D01) to obtain a distance in any direction given $E(B-V)$, and the X-ray quantile colorcolor diagram (QCCD) technique for spectral analysis of lowcount X-ray point sources. In $\S 4$ we give results for X-ray and optical source properties and present our detected candidate CVs and observed stellar X-ray luminosity function. In $\S 5$ we discuss the likely composition of our stellar coronal source population and compare the CV sample to predictions of a simple Galactic distribution model. Finally, in $\S 6$ we present our conclusions.

\section{OBSERVATIONS AND DATA SETS}

\subsection{X-Ray Data Set}

Hong et al. (2005) and J. Hong et al. (2008, in preparation) describe in detail our pipeline processing of archival Chandra observations for use in the ChaMPlane survey and subsequent, more detailed analysis. In summary, source lists from detections in broad $\left(B_{\mathrm{X}}, 0.3-8.0 \mathrm{keV}\right)$, soft $\left(S_{\mathrm{X}}, 0.3-2.5 \mathrm{keV}\right)$, and hard $\left(H_{\mathrm{X}}, 2.5-8.0 \mathrm{keV}\right)$ energy bands are cross-correlated to form a master source list. Source properties such as flux and energy quantiles (see $\S 3.1$ ) are derived in energy bands more appropriate to analysis of low-count sources. These conventional bands are defined as follows: $S_{C}, 0.5-2.0 \mathrm{keV} ; H_{C}, 2.0-8.0 \mathrm{keV}$; and $B_{C}$, $0.5-8.0 \mathrm{keV}$. In the following analysis we consider only X-ray sources of level 1 and above - these being sources unaffected by hot pixels, bad columns, or bad bias values on the ACIS detector, or readout streaks from bright sources, as well as sources too close to the chip boundary; see Hong et al. (2005) for a complete 
TABLE 1

X-Ray Observations Used in this Paper

\begin{tabular}{|c|c|c|c|c|c|c|c|}
\hline \multirow[b]{2}{*}{ OBs. ID } & \multirow[b]{2}{*}{ FIELD NAME } & \multicolumn{2}{|c|}{ Aim PoInT } & \multirow[b]{2}{*}{$\begin{array}{c}\text { No. of Sources } \\
B_{\mathrm{X}}\end{array}$} & \multirow[b]{2}{*}{$\begin{array}{l}\text { EXPOSURE } \\
(\mathrm{ks})\end{array}$} & \multirow[b]{2}{*}{$\begin{array}{c}\text { EFFECTIVE EXP. } \\
(\mathrm{ks})\end{array}$} & \multirow[b]{2}{*}{$\begin{array}{c}N_{22}{ }^{\mathrm{b}} \\
\left(\mathrm{cm}^{-2}\right)\end{array}$} \\
\hline & & $\begin{array}{c}l \\
(\operatorname{deg})\end{array}$ & $\begin{array}{c}b \\
\text { (deg) }\end{array}$ & & & & \\
\hline 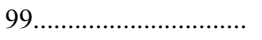 & GRO J1655-40 (J1655) & 344.98178 & 2.45612 & 137 & 43.0 & 42.3 & 1.0 \\
\hline $737 \ldots \ldots \ldots \ldots \ldots \ldots \ldots$ & $\mathrm{G} 347.5-0.5 \mathrm{~b}(\mathrm{G} 347 \mathrm{~b})$ & 347.36606 & -0.85734 & 108 & 40.0 & 38.2 & 3.3 \\
\hline $944 \ldots \ldots \ldots \ldots \ldots \ldots \ldots \ldots \ldots$ & SgrB2 & 0.58834 & -0.02491 & 369 & 100.0 & 96.8 & 10.8 \\
\hline 945 & Gal. center arc (GalCA) & 0.14055 & -0.09707 & 222 & 50.0 & 48.5 & 10.8 \\
\hline $53392^{\mathrm{c}}$ & SgrA $\star$ & 359.94415 & -0.04594 & 2982 & 748.0 & 698.0 & 4.3 \\
\hline
\end{tabular}

a Number of valid (level 1) sources found in the $B_{\mathrm{X}}$ band.

${ }^{\mathrm{b}}$ Integrated column density $N_{\mathrm{H}}$ in units of $10^{22}$ up to a distance of $8 \mathrm{kpc}$, from D01.

c ObsID 53392 is a number we assign internally to refer to our stacked Sgr A* observation.

description of the different levels assigned to sources in our $\mathrm{X}$-ray catalog.

X-ray data for this paper come from three ACIS-I and one ACIS-S Chandra pointings. In addition, we have stacked 14 ACIS-I observations centered on Sgr A* to create a deep image of the GC region. This stack includes the observations analyzed by Muno et al. (2003; with the exception of ObsID 1561), that amount to a total of $590 \mathrm{ks}$ exposure time, as well as ObsIDs 3549, 4683, 4684 , and 5360 . The resulting total exposure time is 748 or $698 \mathrm{ks}$ of good time after processing. The process of dealing with duplicate sources between individual pointings in the stack is detailed in Hong et al. (2005). The final stacked Sgr A* observation we label ObsID 53392. It overlaps partially with ObsID 945-the two share 46 X-ray sources in common. All fields listed in Table 1 were observed with Chandra ACIS-I except J1655, for which ACIS-S was used. Exposure times are given before and after correcting for good time intervals (GTIs).

\subsection{Optical Data Set}

Targets for optical spectral follow-up were selected following observations made in 2000 March with the Mosaic camera on the CTIO $4 \mathrm{~m}$ telescope in $V, R, I$, and $\mathrm{H} \alpha$ filters to identify candidate counterparts as follows (see Zhao et al. 2005 for details). We determine the systematic offsets between the Chandra and Mosaic astrometries, i.e., the boresight correction, for each pair of X-ray and optical images using the iterative procedure described in Zhao et al. (2005). After applying the boresight correction, we search for candidate optical counterparts within some confidence radius of each X-ray source, taking into account optical and X-ray astrometric uncertainties and the boresight error. We elected to search within a $2 \sigma$ radius of each source (thus losing on average $\sim 5 \%$ of the real counterparts). Table 2 summarizes these results. The resulting catalog of candidate counterparts was used to make a target list for the observing runs in 2001-2002 with the Low Dispersion Survey Spectrograph 2(LDSS2) on the $6.5 \mathrm{~m}$ Baade Magellan telescope.

The LDSS2 instrument uses a multiaperture mask with a $\sim 5^{\prime}$ diameter field of view. With a dispersion of $5.3 \AA$ per pixel, we obtained spectra centered on $6500 \AA$ covering 3500-9500 at a resolution of $13.3 \AA$. Masks for LDSS2 were generated with the ldss 2 mask. $f$ FORTRAN code. Using slit lengths between $\sim 5^{\prime \prime}$ and $10^{\prime \prime}$, and three or four alignment stars, between six and $\sim 20$ targets were assigned to each mask. Given the ChaMPlane goal of finding X-ray binaries largely powered by accretion processes, highest priority for inclusion of targets on the masks went to objects showing both X-ray and $\mathrm{H} \alpha$ emission (i.e., $\mathrm{H} \alpha-$ $R<-0.3$ ), followed by X-ray source candidate optical counterparts (regardless of X-ray or optical colors), then $\mathrm{H} \alpha$-bright objects ( $\mathrm{H} \alpha-R<-0.3$ ), and then "marginal" $\mathrm{H} \alpha$ objects (with $-0.3<$ $\mathrm{H} \alpha-R<-0.2)$. Each of these groups was sorted in order of $R$-magnitude brightness, with the brightest objects having highest priority. Details of the observations are given in Table 3.

Data from LDSS2 were reduced using the standard IRAF procedure ccdproc. Spectra were extracted one-by-one using the IRAF doslit package. When crowding in dense fields resulted in multiple stars falling on a slit, the correct target star was identified for extraction by comparing the dispersed CCD image of the spectra with the Mosaic image of the field and a reference image of the sky taken without the LDSS2 slit mask and grism in place. Stars too poorly exposed to find any trace on the CCD, too badly saturated, or on incorrectly cut slits were not extracted (these three cases account for $\sim 20 \%$ of the original targets). In total we extracted 136 usable targets within the five Chandra fields of view. Flux calibration was performed on each extracted spectrum using IRAF routines with flux standard spectra taken each night. Where possible, all extracted spectra were then assigned a spectral classification by visual inspection and comparison with published atlases of optical and near-IR spectra (Torres-Dodgen \& Weaver 1993; Jacoby et al. 1984; Carquillat et al. 1997; Andrillat et al. 1995; Silva \& Cornell 1992).

\section{ANALYSIS}

\subsection{Extinction and Hydrogen Column Density}

To estimate the hydrogen column density $N_{\mathrm{H}}$ for all X-rayoptical matches observed with LDSS2, we use the flux-calibrated optical spectrum of each candidate optical counterpart. We fit the

TABLE 2

Spectral Observations with LDSS2/Magellan $6.5 \mathrm{~m}$

\begin{tabular}{|c|c|c|c|}
\hline Date & Fields Observed & Flux Standards Used & Grism/Filter ${ }^{\mathrm{a}}$ \\
\hline 2001 May $18-20 .$. & SgrA & LTT3864, LTT7987 & MedRed/S2 \\
\hline 2001 July $25-27 \ldots \ldots \ldots \ldots \ldots \ldots$ & SgrB2 & LTT9239, Feige110, LTT7379 & MedBlue/None \\
\hline 2002 June $16-19 \ldots \ldots \ldots \ldots \ldots \ldots$ & J1655, GalCA, SgrA, SgrB2, G347b & LTT9239, EG274 & MedBlue/None \\
\hline
\end{tabular}

${ }^{\text {a }}$ Second-order blocking filter. 
TABLE 3

X-Ray-to-Optical Matching Summary

\begin{tabular}{|c|c|c|c|c|c|}
\hline Field Name & $N(\mathrm{X})^{\mathrm{a}}$ & $N(\mathrm{Xmatch})^{\mathrm{b}}$ & $N(\mathrm{Opt})^{\mathrm{c}}$ & $N(\text { Spectra })^{\mathrm{d}}$ & $N(\text { Id. Spec. })^{\mathrm{e}}$ \\
\hline 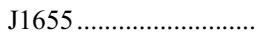 & 137 & 58 & 227 & 32 & 28 \\
\hline G347b......................... & 108 & 53 & 147 & 26 & 25 \\
\hline 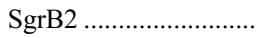 & 369 & 112 & 165 & 52 & 45 \\
\hline 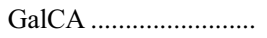 & 222 & 48 & 67 & 9 & 9 \\
\hline 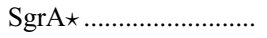 & 2982 & 327 & 340 & 17 & 15 \\
\hline Total ............................ & 3818 & 598 & 946 & 136 & 122 \\
\hline
\end{tabular}

a The number of unique (level 1) X-ray sources in all bands.

b The number of X-ray sources that have any optical counterpart(s).

c The number of optical sources falling inside $2 \sigma$ error circles. Some X-ray sources match multiple optical sources.

d The number of optical spectra of Chandra matches obtained with LDSS2.

e The number of identifiable spectra from this sample.

spectrum with a blackbody attenuated by interstellar reddening, using the analytical parameterization of the average Galactic extinction law $E(\lambda-V) / E(B-V)$ given by Howarth (1983). We limit the region to be fitted to the central part of the spectra, 5500$6700 \AA$, in part to simplify the fitting procedure, and also because the flux-calibrated spectra anomalously fall off in flux redward of $\sim 7000 \AA$. We also interpolate over major spectral lines at 5575, 6300 , and $6562 \AA$ and in the case of the molecular bands present in $\mathrm{M}$ type stars we fit a smooth continuum shape to the star at 34 points between bands (we use a Legendre polynomial of order $10)$ and fit to this instead. Using a fixed effective temperature ( $\left.T_{\text {eff }}\right)$, estimated from our spectral type determination from spectral lines, we fit for $E(B-V)$ and a normalization factor: $R^{2} / d^{2}$ (radius of object $R$, distance $d$ ). We obtain $A_{V}$ from the standard relation $A_{V}=3.1 E(B-V)$ and the hydrogen column density from $N_{\mathrm{H}}=1.79 \times 10^{21} \times A_{V} \mathrm{~cm}^{-2}$ (from Predehl \& Schmitt 1995). The $T_{\text {eff }}$ in each case is representative of the spectral type range we were able to estimate given only visual classification of each spectrum and no constraint on luminosity class (i.e., A/F means A8-F2, luminosity class undetermined). The fit is independent of any X-ray emission properties. Effective temperatures and adopted photometry for each spectral type "bin" are given in Table 4 (see references listed in the table). Example fits for a $\mathrm{K}$ and an M (polynomial fit) star are shown in Figure 1.

To test the above, we derive comparison $N_{\mathrm{H}}$ values using our $V, R$, and $I$ photometry (where available). We calculate $E(V-R) \equiv V-R-(V-R)_{0}$ and $E(R-I) \equiv R-I-(R-I)_{0}$, selecting appropriate values for the intrinsic $(V-R)_{0}$ and $(R-I)_{0}$ colors (from Cox 2000) for a given spectral type (see Table 4). From Dopita \& Sutherland (2003) we find $A_{V}=3.97 E(V-R)$ and $A_{V}=3.76 E(R-I)$ and we convert to $N_{\mathrm{H}}$ as above. Figure 2 shows spectral fit-determined values of $N_{\mathrm{H}}$ against the colordetermined results. The values are correlated somewhat, but the spectral fit $N_{\mathrm{H}}$ is systematically higher than that from photometry: from a simple linear regression test we find $N_{\mathrm{H}}$ (fit) $=0.96 \pm$ $0.09\left[N_{\mathrm{H}}(R-I)\right]+0.2$ and $N_{\mathrm{H}}($ fit $)=0.81 \pm 0.07\left[N_{\mathrm{H}}(V-R)\right]+$ 0.25 . Dropping the five highest points in either plot, however, worsens the correlation: $N_{\mathrm{H}}(\mathrm{fit})=0.81 \pm 0.13\left[N_{\mathrm{H}}(R-I)\right]+$ 0.27 and $N_{\mathrm{H}}$ (fit) $=0.65 \pm 0.1\left[N_{\mathrm{H}}(V-R)\right]+0.3$. This overestimate in $N_{\mathrm{H}}$ is also seen in a color-magnitude diagram for our stellar sources (Figure 3) of absolute visual magnitude $M_{V}$ against dereddened color $V-R$. A comparison main sequence is plotted using the data from Table 4 . We estimate the systematic excess in $A_{V}$ from our fitting technique to be 1.1 (equivalent to $N_{\mathrm{H}}$ of $2 \times 10^{21} \mathrm{~cm}^{-2}$ ). This overestimate is perhaps due to the inadequacy of the blackbody spectrum as a fit to late-type stellar spectra over this wavelength range where many absorption lines significantly modify the continuum shape.

\subsection{Quantile Analysis}

For stars with low signal-to-noise ratio optical spectra we assign in Table 5 a nonclassification to the object, "?". In order to place constraints on $N_{\mathrm{H}}$ for these sources, and for the CVs, whose optical spectra are not well modeled by single-temperature blackbodies, we utilize X-ray quantile analysis. Objects for which this has been carried out are marked with a dagger in Table 5. Quantile analysis was first presented in Hong et al. (2004, hereafter H04). It allows us to derive X-ray spectral information despite low source counts. It involves placing sources on an X-ray color-color diagram by the median and quartile energy fractions of their source counts. As defined by $\mathrm{H} 04$, any general quantile $Q_{x}$ is calculated as

$$
Q_{x}=\frac{E_{x \%}-E_{\mathrm{lo}}}{E_{\mathrm{up}}-E_{\mathrm{lo}}},
$$

TABLE 4

Effective Temperature Assignment and Photometric Properties Used

\begin{tabular}{|c|c|c|c|c|c|}
\hline Classification & Sp. Type Range & $\begin{array}{l}T_{\text {eff }} \\
(\mathrm{K})\end{array}$ & $V-R$ & $R-I$ & $M_{V}$ \\
\hline Early A.... & $\mathrm{A} 2-\mathrm{A} 4$ & 9100 & 0.08 & 0.03 & $1.8 \pm 0.4$ \\
\hline Mid A....... & $\mathrm{A} 4-\mathrm{A} 6$ & 8500 & 0.16 & .06 & $2.0 \pm 0.5$ \\
\hline Late $\mathrm{A} \ldots .$. & $\mathrm{A} 6-\mathrm{A} 8$ & 7700 & 0.19 & 0.09 & $2.4 \pm 0.6$ \\
\hline A/F & $\mathrm{A} 8-\mathrm{F} 2$ & 7200 & 0.30 & 0.17 & $2.8 \pm 0.6$ \\
\hline 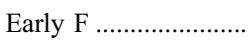 & $\mathrm{F} 2-\mathrm{F} 4$ & 6750 & 0.35 & 0.20 & $3.2 \pm 0.4$ \\
\hline 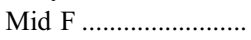 & F4-F6 & 6500 & 0.40 & 0.24 & $3.5 \pm 0.4$ \\
\hline Late F & F6-F8 & 6200 & 0.47 & 0.29 & $3.8 \pm 0.4$ \\
\hline F/G & $\mathrm{F} 8-\mathrm{G} 2$ & 5950 & 0.50 & 0.31 & $4.3 \pm 0.7$ \\
\hline Early G.......................... & G2-G4 & 5600 & 0.53 & 0.33 & $4.6 \pm 0.4$ \\
\hline Mid G......... & G4-G6 & 5400 & 0.60 & 0.42 & $4.9 \pm 0.5$ \\
\hline Late G .......................... & G6-G8 & 5200 & 0.64 & 0.43 & $5.3 \pm 0.5$ \\
\hline $\mathrm{G} / \mathrm{K}$ & $\mathrm{G} 8-\mathrm{K} 2$ & 5050 & 0.70 & 0.48 & $5.8 \pm 1.0$ \\
\hline 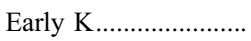 & $\mathrm{K} 2-\mathrm{K} 4$ & 4700 & 0.80 & 0.53 & $6.7 \pm 0.6$ \\
\hline Mid K........ & $\mathrm{K} 4-\mathrm{K} 6$ & 4300 & 1.10 & 0.75 & $7.4 \pm 0.6$ \\
\hline Late $\mathrm{K} .$. & $\mathrm{K} 6-\mathrm{K} 8$ & 4000 & 1.15 & 0.78 & $8.4 \pm 0.6$ \\
\hline $\mathrm{K} / \mathrm{M} \ldots \ldots \ldots \ldots$ & K8-M2 & 3700 & 1.25 & 0.93 & $9.3 \pm 1.0$ \\
\hline & & & 1.42 & 1.15 & $11.1 \pm 1.6$ \\
\hline Mid M & M4-M6 & 3000 & 1.8 & 1.67 & $13.2 \pm 2.0$ \\
\hline
\end{tabular}

NotEs.-References for colors, temperatures, and absolute magnitudes: Johnson (1966), Bessell (1991), Mikami \& Heck (1982), Houk et al. (1997), Gray (1992), and Cox (2000). We estimate an uncertainty in the assigned $T_{\text {eff }}$ values of $\pm 500 \mathrm{~K}$, based on the spread of values found in the various references. 

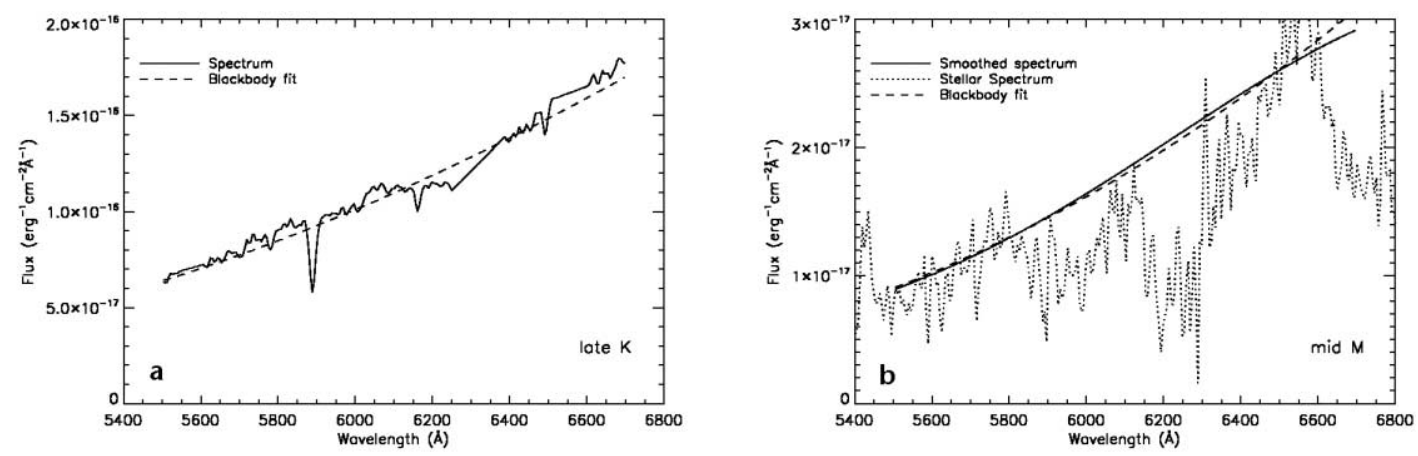

FIG. 1.- Two example fits to spectra from the LDSS2 sample. The panel for the M star (right) also shows the polynomial fit to the spectrum (see text for description) to which the blackbody curve was subsequently fit.
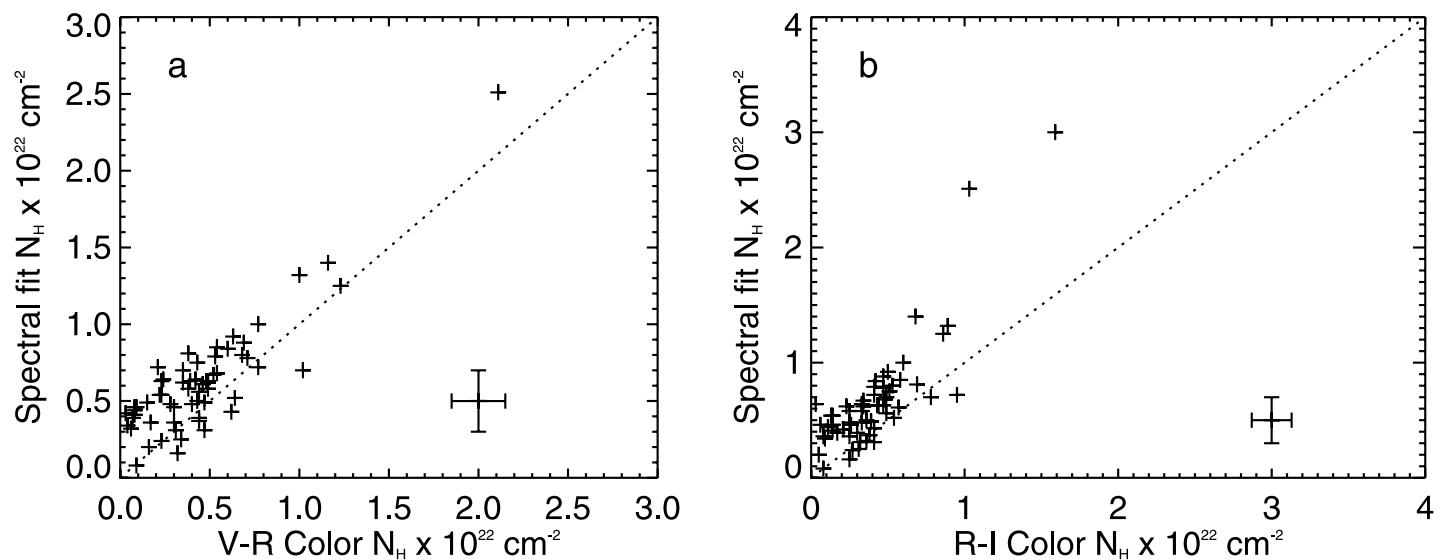

Fig. 2.-Plots of hydrogen column density for all stars with available $V-R$ or $R-I$ photometry in the sample, derived from the spectral fit and color methods detailed in $\S \S 3.1$ and 3.2. Typical error bars and lines of $N_{\mathrm{H}}($ color $)=N_{\mathrm{H}}($ fit) (dotted line) are shown for reference.

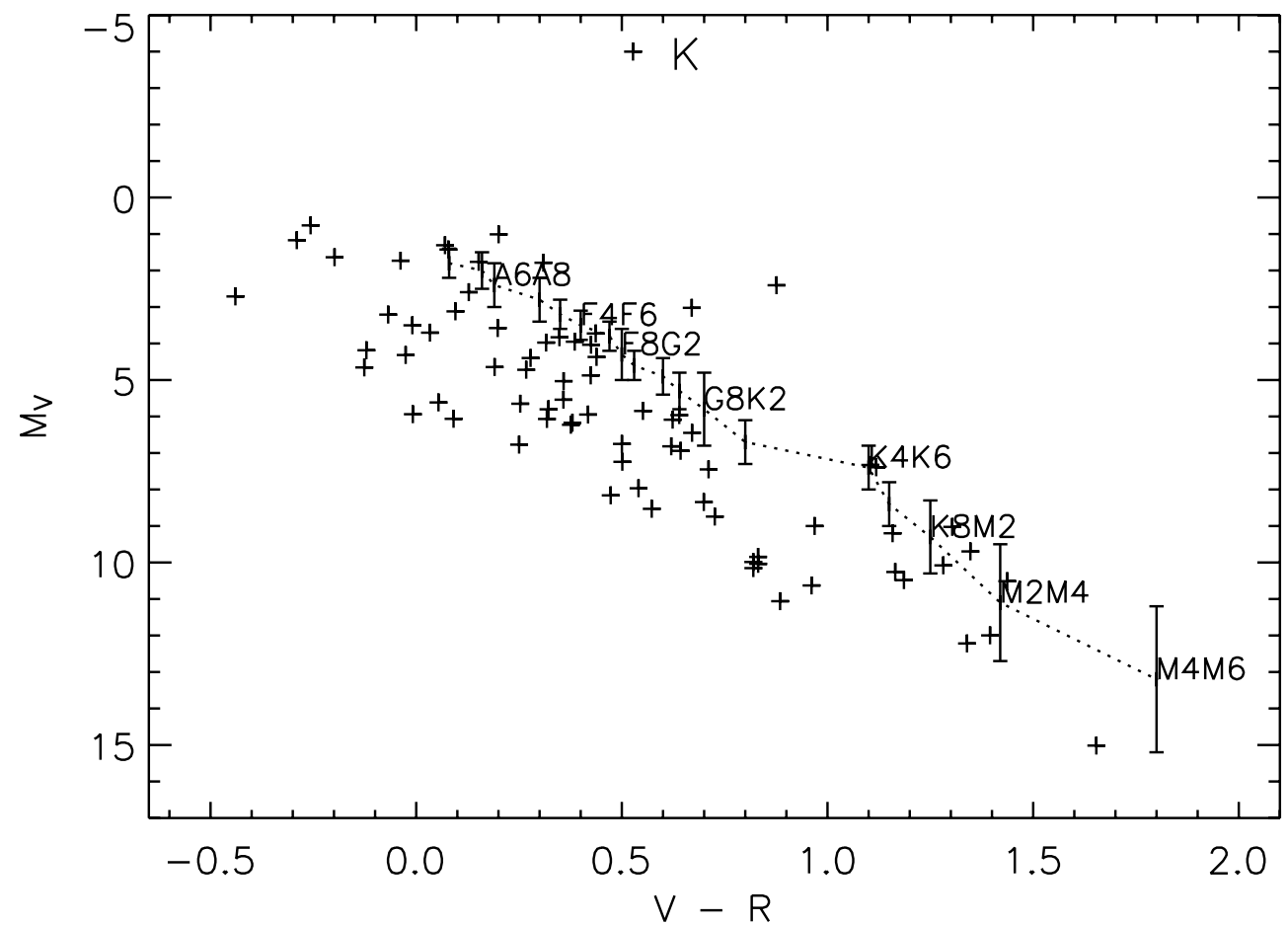

Fig. 3. - Color-magnitude diagram of the stars in the LDSS2 sample. We plot $M_{V}=V-5 \log ($ dist $)+5-A_{V}$, and $(V-R)_{0}=V-R-0.781 E(B-V)$. The star marked " $\mathrm{K}$ " is the K giant in SgrB2, ChOPS J174642.22-282907.4. The dotted curve and spectral type labels are from Cox (2000) and the error bars show the range of $M_{V}$ for spectral type range plotted. 
TABLE 5

Combined Results for $2 \sigma$ Optical Matches

\begin{tabular}{|c|c|c|c|c|c|c|c|c|c|c|c|c|c|}
\hline $\begin{array}{c}\text { ChOPS ID } \\
\text { (1) }\end{array}$ & $\begin{array}{l}\text { Type }^{\mathrm{b}} \\
\text { (2) }\end{array}$ & $\begin{array}{l}\text { Special }^{\mathrm{c}} \\
\text { (3) }\end{array}$ & $\begin{array}{l}\text { Counts }^{\mathrm{d}} \\
\text { (4) }\end{array}$ & $\begin{array}{c}N_{22}{ }^{\mathrm{e}} \\
(5)\end{array}$ & $\begin{array}{c}\log \left(F_{\mathrm{X}} / F_{R}\right)^{\mathrm{f}} \\
(6)\end{array}$ & $\begin{array}{l}\text { Dist. }^{\mathrm{g}} \\
\text { (7) }\end{array}$ & $\begin{array}{r}M_{V} \\
(8)\end{array}$ & $\begin{array}{c}\log \left(L_{X}\right)^{\mathrm{h}} \\
\quad(9)\end{array}$ & $\begin{array}{c}R \\
(10)\end{array}$ & $\begin{array}{l}N_{M}{ }^{\mathrm{i}} \\
(11)\end{array}$ & $\begin{array}{l}\operatorname{Srch}^{\mathrm{j}} \\
(12)\end{array}$ & $\begin{array}{l}\text { Offset }^{j} \\
(13)\end{array}$ & $\begin{array}{l}P_{\mathrm{Rn}}{ }^{\mathrm{j}} \\
(14)\end{array}$ \\
\hline \multicolumn{14}{|c|}{$\mathrm{J} 1655$} \\
\hline J165343.19-394826.1 ................ & $\mathrm{G} / \mathrm{K}$ & $\ldots$ & $11 \pm 5$ & $0.3(1)$ & $-2.5 \pm 0.4$ & 1.4 & $5.7(9)$ & $29.54 \pm 0.7$ & $17.48(1)$ & 1 & 1.199 & 0.420 & 0.207 \\
\hline $\mathrm{J} 165350.37-394621.9 \ldots \ldots \ldots \ldots \ldots$ & Mid M dMe? & $\ldots$ & $12 \pm 5$ & $0.2(2)$ & $-1.8 \pm 0.5$ & 1.4 & $8(2)$ & $29.43 \pm 0.8$ & $19.32(1)$ & 2 & 1.886 & 1.271 & 0.712 \\
\hline J165350.70-395339.6 ……........ & Early K & $\ldots$ & $8 \pm 5$ & $0.3(1)$ & $-2.2 \pm 0.4$ & 1.5 & $6.8(9)$ & $29.46 \pm 0.7$ & $18.44(1)$ & 1 & 0.858 & 0.788 & 0.082 \\
\hline J165351.90-394812.7 ……........ & F/G? & by & $18 \pm 6$ & $0.7(2)$ & $-1.8 \pm 0.6$ & 4.0 & $5(2)$ & $30.59 \pm 0.8$ & $20.63(1)$ & 1 & 0.715 & 0.692 & 0.064 \\
\hline J165354.54-394741.0 ............... & $\mathrm{G} ?$ & rs & $11 \pm 5$ & $0.9(2)$ & $-3.5 \pm 0.7$ & 4.5 & $1(1)$ & $30.38 \pm 5.9$ & $17.96(1)$ & 1 & 0.874 & 0.322 & 0.092 \\
\hline J165356.10-394732.7 ................. & Late $\mathrm{F}$ & $\ldots$ & $9 \pm 5^{*}$ & $0.4(2)$ & $-3.1 \pm 0.5$ & 1.9 & $\ldots$ & $29.87 \pm 0.7$ & $16.30(1)$ & 1 & 0.933 & 0.391 & 0.113 \\
\hline J165356.98-394636.7 ……........ & Early G & $\ldots$ & $8 \pm 5^{*}$ & $0.9(2)$ & $-2.8 \pm 0.6$ & 4.8 & $2(2)$ & $30.81 \pm 5.4$ & 19.10(3) & 1 & 1.322 & 0.358 & 0.271 \\
\hline J165357.34-394847.9 …........... & Mid M dMe? & $\ldots$ & $18 \pm 5$ & $0.2(1)$ & $-1.3 \pm 0.4$ & 1.1 & $9.9(9)$ & $29.43 \pm 0.7$ & 19.93(2) & 1 & 0.677 & 0.380 & 0.050 \\
\hline J165357.85-395032.1 ….............. & F/G? & $\ldots$ & $6 \pm 4^{*}$ & $0.6(1)$ & $-2.5 \pm 0.5$ & 3.1 & $4.1(9)$ & $30.12 \pm 0.8$ & $19.20(1)$ & 1 & 0.584 & 0.230 & 0.024 \\
\hline J165358.28-394812.6 ……........ & Mid M & $\ldots$ & $6 \pm 4^{*}$ & $0.1(1)$ & $-2.1 \pm 0.5$ & 0.8 & $10.0(8)$ & $28.50 \pm 0.8$ & $19.25(1)$ & 1 & 1.045 & 0.510 & 0.163 \\
\hline
\end{tabular}

Notes.-Table 5 is published in its entirety in the electronic edition of the Astrophysical Journal. A portion is shown here for guidance regarding its form and content

a ChaMPlane IDs have prefix ChOPS

${ }^{b}$ See Table 4 for explanation of spectral type classification.

Additional classification of source type: q, qLMXB candidate; by, BY Dra candidate; rs, RS CVn candidate; tt, T Tauri; cv, CV.

${ }^{\mathrm{d}}$ Background-subtracted net counts in the $B_{\mathrm{X}}$ band $(0.3-8.0 \mathrm{keV})$. Asterisks indicate sources that have $\mathrm{S} / \mathrm{N}<3$.

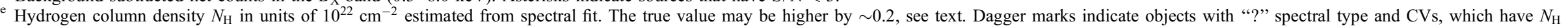
estimated from QCCD analysis.

${ }^{\mathrm{f}}$ Logarithm of the ratio of unabsorbed flux in the $S_{C}$ band $0.5-2.0 \mathrm{keV}$ to unreddened optical $R$-band flux [ergs $\mathrm{cm}^{-2} \mathrm{~s}^{-1}(1000 \AA)^{-1}$ ].

The distance in kiloparsecs. Error $\approx 60 \%$

Logarithm of X-ray luminosity in the $S_{C}$ band $\left(\mathrm{ergs} \mathrm{s}^{-1}\right.$ )

Number of optical matches found within the $95 \%$ confidence $(2 \sigma)$ error circle of this X-ray source.

"Srch": the combined $95 \%$ X-ray and optical position error circle $(\operatorname{arcsec})$ used to search for optical matches. "Offset": the positional offset in arcsec between X-ray and optical position. " $P_{\mathrm{Rn}}$ ": random match probability given error circle size and measured local optical projected surface density. 

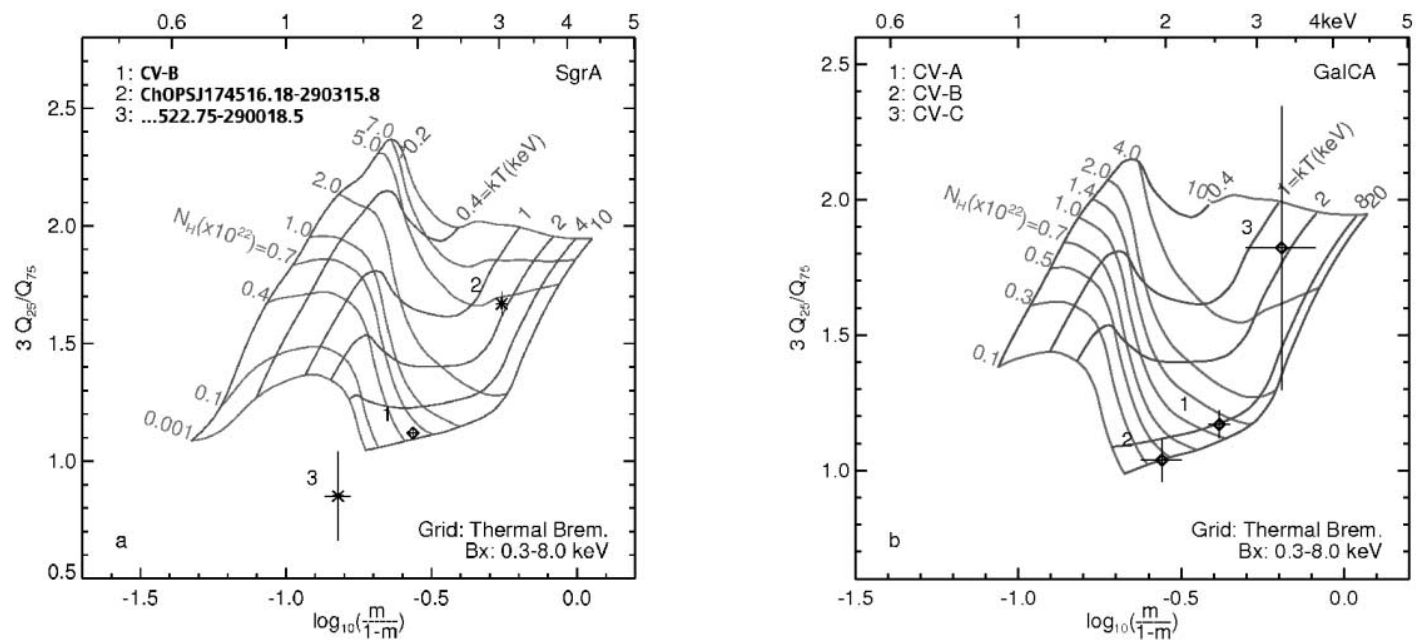

FIG. 4.-Left: QCCD plot for ObsID 53392 (SgrA $\star$ ) showing the positions of CV-B and nonidentified sources from the SgrA $\star$ field with at least 107 counts. Right: ObsID 945 (GalCA) showing all three CVs. [See the electronic edition of the Journal for a color version of this figure.]

where $E_{x \%}$ is the energy below which the net counts is $x \%$ of the total number of counts between $E_{\mathrm{lo}}$ and $E_{\mathrm{up}}$; we select $E_{\mathrm{lo}}=0.3$ and $E_{\text {up }}=8.0 \mathrm{keV}\left(B_{\mathrm{X}}\right.$ band $)$ for our analysis. We plot the ratio $Q_{25} / Q_{75}$ against $\log _{10}[m /(1-m)]\left(m\left[\equiv Q_{50}\right]\right.$ is the median). For a given spectral model, we overlay a grid of column density $N_{\mathrm{H}}$ and model parameter - we interpolate to find a value for $N_{\mathrm{H}}$. The grid shape is dependent on the Chandra ACIS response function for the ObsID considered.

Figure 4 shows QCCD plots for ObsID 53392, which includes CV-B (detected with $\sim 3500$ counts in this ObsID) and ObsID 945, which includes all three CVs we have detected in the Chandra fields considered in this paper. We use a thermal bremsstrahlung model to construct the grids shown in this case.

\subsection{Extinction Model for the Galaxy}

We require a method of deriving luminosity for our sources and so need a way of estimating their distances. Recently, D01 constructed a three-component model for the dust distribution in the Galaxy. This is used via a FORTRAN code (Drimmel et al. 2003, hereafter D03) to derive the extinction $A_{V}$ as a function of distance from the Sun over the whole sky. The spatial resolution on which values of $A_{V}$ can be measured is $\sim 20^{\prime}$. Figure 5 shows the run of $A_{V}$ with distance for each field in this paper. After fitting the optical spectra or using quantile analysis to derive extinction values $A_{V}$, we utilize the model of D01 to derive distances to all sources in this paper. The values listed in Table 5 are not corrected for the overestimate in $A_{V}$. As a check on the results, the spectral fit scaling factor $R^{2} / d^{2}$ allows us to derive a radius which we compare with expected values for stars of given spectral type and $M_{V}$ (e.g., Cox 2000).

The code has a "rescaling" option to account for small-scale variations and clumping in the dust density that are smoothed over by the model. This is based on a factor dependent on the residuals between the $C O B E$ observed flux at $240 \mu \mathrm{m}$ and the model prediction for the same. It was found that for all fields, using the rescaling option in the D03 code worked well in producing reasonable stellar radii within $50 \%$ of tabulated values (e.g., Cox 2000), with the exception of G347b, where radii were systematically a factor of 4-5 times higher.

For G347b we derive $A_{V}(d)$ using extracted emission spectra of Galactic molecular CO (from the survey of Dame et al. 2001) and $21 \mathrm{~cm} \mathrm{H}$ i diffuse gas (data taken from the Southern Galactic Plane Survey; Taylor et al. 2003) to derive the column density of molecular and atomic hydroge, $\mathrm{n}$ respectively, thus $N_{\mathrm{H}}$ via $N_{\mathrm{H}}=$ $N_{\mathrm{H}_{\mathrm{I}}}+N_{\mathrm{H}_{2}}$ and so $A_{V}$. The $\mathrm{H}$ I data have a resolution of $\sim 1^{\prime}$, but for this analysis were smoothed to $3^{\prime}$ resolution with $2.7^{\prime}$ spacing. Emission from $\mathrm{CO}$ was assigned a distance based on line-of-sight velocity, splitting the near/far ambiguity based on the latitude of observation, and using the Galactic rotation curve of Brand \& Blitz (1993). We assumed a FWHM layer thickness for $\mathrm{H}$ i of 220 pc (Dickey \& Lockman 1990), and for CO of 120 pc (Dame et al. 2001). Emission beyond the terminal velocity cutoff was redistributed in a Gaussian below the cutoff with the Gaussian dispersion equal to the cloud-cloud velocity dispersion: $\sigma(\mathrm{CO})=$ $4 \mathrm{~km} \mathrm{~s}^{-1}, \sigma(\mathrm{H} \mathrm{I})=8 \mathrm{~km} \mathrm{~s}^{-1}$. We assume an atomic hydrogen spin temperature of $140 \mathrm{~K}$. We performed this calculation along

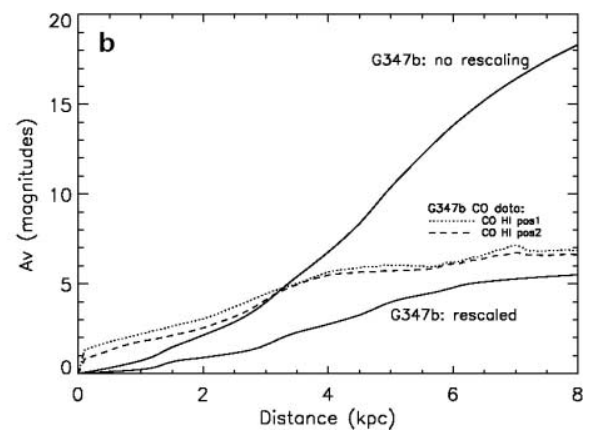

FIG. 5.-Plots of $A_{V}$ (rescaled values) vs. distance from the model of D01 for the five Chandra fields. For field G347b (right) we show the results of both rescaled and nonrescaled $A_{V}$ vs. distance from this paper, and also overplot our own results, as derived from CO+H I observations. See $\S 3.2$ for a description. 
two lines of sight - in Galactic coordinates these are at $(l, b)$ $347.375,-0.75$ and $347.375,-0.875$ (positions 1 and 2, respectively). Figure 5 (right) shows the resulting $A_{V}$ versus distance plots for field G347b, labeled position 1 and position 2 . We were unable to perform the same derivation for the other fields as the three Galactic center fields (SgrA $\star$, SgrB2, and GalCA) are too close to the Galactic plane to easily assign distances to molecular emission, and for field $\mathrm{J} 1655$ the $\mathrm{H}$ I data available were not at high enough resolution to accurately run the calculation.

\subsection{Calculating X-Ray Fluxes}

We calculate unabsorbed X-ray fluxes for all sources from their net count rates using Sherpa. ${ }^{4}$ For simplicity, we assume that the $\mathrm{X}$-ray radiation produced by the majority of objects in our sample will be emission from a hot $\left(T>10^{6} \mathrm{~K}, k T>0.1 \mathrm{keV}\right)$ coronal plasma. To calculate fluxes in the hard $\left(H_{C}\right)$ and $\operatorname{soft}\left(S_{C}\right)$ bands for each star in our sample we thus adopt a simple singletemperature MEKAL model ${ }^{5}$ (bremsstrahlung emission of an optically thin, thermal plasma with metal absorption and emission lines; see Mewe et al. 1985) at $1 \mathrm{keV}$ and use the $N_{\mathrm{H}}$ value listed in Table 5. Section 3.4 discusses the uncertainty introduced by our choice of spectral model on our results for X-ray flux. We derive X-ray luminosities via $L_{\mathrm{X}}=4 \pi d^{2} F_{\mathrm{X}} \operatorname{ergs~s}^{-1}$. The X-rayto-optical flux ratio is calculated via

$$
\log \left(F_{\mathrm{X}} / F_{R}\right)=\log \left(F_{\mathrm{X}}\right)+0.4 R+5.765
$$

and

$$
\log \left(F_{\mathrm{X}} / F_{V}\right)=\log \left(F_{\mathrm{X}}\right)+0.4 V+5.426,
$$

where we have assumed a square optical filter transmission function of width $1000 \AA$, centered on the filter's quoted central wavelength, with an underlying A0 stellar spectrum to calculate the constants. Note that the overestimate in $N_{\mathrm{H}}$ from $\S 3$ carried through results in $\Delta \log \left(F_{X} / F_{V}\right)=+0.3$ to +0.5 and $\Delta \log \left(L_{X}\right) \sim$ -0.3 . The exact correction depends on the object's initial $N_{\mathrm{H}}$. This is not incorporated in the results given in Table 5.

\subsection{Error Analysis}

The primary source of error in our analysis is the calculation of $E(B-V)$-this has three components. First, there is the uncertainty in assigned $T_{\text {eff }}$ values $( \pm \sim 500 \mathrm{~K})$. Second, from systematic errors in the flux-calibrated spectra (from the extraction and calibration processes). The flux calibration error ranges from $\sim 10 \%$ to $60 \%$ - estimated by comparing repeat observations of stars observed on multiple nights. This was the case for moderate and also high $\mathrm{S} / \mathrm{N}$ spectra. Variations were evident in spectra both within a night and from night to night. Third, errors introduced by our spectral fitting code. We estimate this by selecting 17 standard star spectra (types M5 V through A7 V) from the catalog of Jacoby et al. (1984), applying a range of fake values of interstellar reddening $[E(B-V)=0.3-4.0]$ with the fmunred command in IDL, ${ }^{6}$ and then attempting to retrieve this fake reddening with our spectral fit code. The "error" on the returned value $\Delta E(B-V) \approx 50 \%$ at $E(B-V) \approx 0.3$, ranging down to $\approx 6 \%$ at $E(B-V) \approx 1.3$ (independent of spectral type). We linearly interpolate this trend to calculate the uncertainty produced

\footnotetext{
4 See http://cxc.harvard.edu/sherpa/threads/index.html.

5 Model: xsmekal in Sherpa.

6 See http://idlastro.gsfc.nasa.gov/.
}

by the fitting process at any $E(B-V)$. The quoted error in column density $N_{\mathrm{H}}$ in Table 5 in column (5) combines all these three sources.

Errors quoted in Table 5 for $\log \left(F_{\mathrm{X}} / F_{R}\right)$ and $\log \left(L_{\mathrm{X}}\right)$ incorporate uncertainty in $N_{\mathrm{H}}$, in the X-ray count rate, and in photometric error in the $R$ magnitude (a relatively small contribution). The choice of X-ray spectral model (1.0 keV MEKAL) also contributes to our uncertainty. Using a power-law model with spectral index $\Gamma=1.7$ or a MEKAL $(k T=2.0 \mathrm{keV})$ reduces the $S_{C}$ band flux by between $10 \%\left(\right.$ at $N_{\mathrm{H}} \approx 0.2 \times 10^{22} \mathrm{~cm}^{-2}$ ) and $50 \%\left(\right.$ at $\left.N_{\mathrm{H}} \approx 2.0 \times 10^{22} \mathrm{~cm}^{-2}\right)$. A MEKAL model with $k T=$ $0.5 \mathrm{keV}$ creates a similar increase in the $S_{C}$ band flux. Thus, the errors quoted in $\log \left(F_{\mathrm{X}} / F_{R}\right)$ and $\log \left(L_{\mathrm{X}}\right)$ should be considered as lower limits.

Using the model of D01 introduces error in our distance calculation. Results for $d$ derived from this model for Galactic longitudes $|l|<20^{\circ}$ are likely to suffer from significant systematic uncertainty, as true structure in the absorbing interstellar medium is significant in this part of the Galaxy and is poorly modeled in the code. The fact that fields SgrB2 and GalCA $\left(\sim 0.5^{\circ}\right.$ apart on the sky) are indistinguishable on this figure and the curve for field $\operatorname{Sgr} A \star$ (which is only $\sim 12^{\prime}$ away) is significantly different is almost certainly a resulting artifact, not a real trait of these two three fields. The combined error from the spectral fit and the dust model (estimated by comparing variation in $A_{V}$ across the Chandra field of view) in the distances we derive is typically $\lesssim 60 \%$. We adopt $60 \%$ as the error on the distances that we quote in Table 5 . This carries through to the error in X-ray luminosity and optical absolute magnitudes.

\section{RESULTS}

Table 5 presents our spectral results. Optical photometry comes from data from our 2000 and 2003 CTIO Mosaic runs (see Zhao et al. 2005). For each source in Table 5 we list the ChaMPlane IAU optical source ID (col. [1]). Readers desiring to find detailed X-ray counterpart properties can readily search our online X-ray database $^{7}$ using the optical source ID for coordinates. We then give the source properties: spectral classification (col. [2]), net X-ray source counts in the $B_{\mathrm{X}}$ band (col. [3]), the hydrogen column density $N_{\mathrm{H}}$ as derived from our fitting technique or quantile analysis (col. [4]), and the unabsorbed X-ray-to- $R$ band flux ratio in the $S_{C}$ band, adopting a $1.0 \mathrm{keV}$ MEKAL X-ray spectral model (col. [5]; see $\S 2$ for band definitions). Although subsequent analysis is performed on a derivation of $\log \left(F_{X} / F_{V}\right)$ for easier comparison with the literature, the actual numbers do not differ significantly from those listed for $\log \left(F_{\mathrm{X}} / F_{R}\right)$. In addition, since $\sim 10 \%$ of our objects are undetected in $V$, we can give a value for more sources by presenting this information instead. The distance and the derived absolute visual magnitude $M_{V}$ are in columns (6) and (7). We then give X-ray luminosity, followed by the optical $R$ magnitude and the number of optical sources found to match the same X-ray position (cols. [8], [9], and [10]). Column (11) gives the X-ray $2 \sigma$ search radius size in arcseconds, and column (12) gives the offset of the optical position in arcseconds from the center of the X-ray error circle. Column (13) gives the expected number of optical sources that should fall in an error circle of this size by chance, given the observed surface density of stars within $1^{\prime}$ of the X-ray position on the Mosaic image. For almost all objects in Table 5 one optical source matches the X-ray position; however, in the J1655 field ChOPS J165407.44-394542.7 and J165350.37-394621.9, in

\footnotetext{
7 See http://hea-www.harvard.edu/ChaMPlane/database_xray.html.
} 
TABLE 6

$\mathrm{H} \alpha-R<-0.3$ ОвЈеСтS

\begin{tabular}{|c|c|c|c|c|}
\hline ChOPS ID & $\mathrm{H} \alpha-R$ & $R$ & Spectrum & $\log \left(F_{\mathrm{X}} / F_{R}\right)$ \\
\hline \multicolumn{5}{|c|}{$\mathrm{J} 1655$} \\
\hline $\begin{array}{l}335.32-393715.9 \ldots \ldots \ldots \ldots \ldots \\
422.01-395205.0 \ldots \ldots \ldots \ldots \ldots . . .\end{array}$ & $\begin{array}{l}-0.46(6) \\
-0.39(2)\end{array}$ & $\begin{array}{l}21.5 \\
19.6\end{array}$ & $\begin{array}{l}\text { None } \\
\text { dMe }\end{array}$ & $\begin{array}{c}<-0.99^{\mathrm{a}} \\
-1.5 \pm 0.5\end{array}$ \\
\hline \multicolumn{5}{|c|}{ GalCA } \\
\hline $\begin{array}{l}638.02-285326.2 \ldots \ldots \ldots \ldots \ldots . . \\
656.89-285233.9 \ldots \ldots \ldots \ldots \ldots . .\end{array}$ & $\begin{array}{l}-0.65(2)^{\mathrm{b}} \\
-0.19(4)^{\mathrm{c}}\end{array}$ & $\begin{array}{l}20.2 \\
21.3\end{array}$ & $\begin{array}{l}\mathrm{CV}(\mathrm{A}) \\
\mathrm{CV}(\mathrm{C})\end{array}$ & $\begin{array}{l}1.2 \pm 0.6 \\
<-2.3\end{array}$ \\
\hline \multicolumn{5}{|c|}{ SgrB2 } \\
\hline $\begin{array}{l}732.09-282033.8 \ldots \ldots \ldots \ldots \ldots . . . \\
708.21-282724.8 \ldots \ldots \ldots \ldots \ldots . . .\end{array}$ & $\begin{array}{l}-0.61(1) \\
-0.38(1)\end{array}$ & $\begin{array}{l}16.2 \\
19.8\end{array}$ & $\begin{array}{l}\text { T Tauri } \\
\text { G? }\end{array}$ & $\begin{array}{l}-2.7 \pm 0.5 \\
-2.5 \pm 0.5\end{array}$ \\
\hline \multicolumn{5}{|c|}{ SgrA } \\
\hline $\begin{array}{l}511.51-290236.8 \ldots \ldots \ldots \ldots \ldots \\
559.18-290418.9 \ldots \ldots \ldots \ldots \ldots . \\
607.52-285951.3 \ldots \ldots \ldots \ldots \ldots .\end{array}$ & $\begin{array}{r}-0.31(1) \\
-0.6(1) \\
-0.61(4)\end{array}$ & $\begin{array}{l}19.2 \\
22.7 \\
21.8\end{array}$ & $\begin{array}{l}\mathrm{dMe} \\
\text { None } \\
\mathrm{CV}(\mathrm{B})\end{array}$ & $\begin{array}{l}-2.5 \pm 0.9 \\
-1.5 \pm 0.5 \\
-0.1 \pm 0.9\end{array}$ \\
\hline
\end{tabular}

Note. - Numbers in parentheses with the $\mathrm{H} \alpha-R$ value represent the error in last quoted digit.

${ }^{\text {a }}$ Undetected in the $S_{C}$ band-estimated upper limit to flux ratio.

b This value of $\mathrm{H} \alpha-R$ was recorded in 2000. In 2003 it had dropped to -0.182 .

${ }^{\mathrm{c}} \mathrm{CV}-\mathrm{C}$ is included here despite having $\mathrm{H} \alpha-R>-0.3$.

the G347b field ChOPS J171524.21-395950.9, and in the SgrB2 field ChOPS J174719.59-282204.9, J174633.29-282512.7, and J174634.65-282721.1 are all one of multiple matches to single X-ray sources. In the case of ChOPS J165350.37-394621.9 in J1655 we have a spectrum of the other match - we select the M-type star as the more likely X-ray-emitting candidate over the G-type alternate. The "Type" column follows this scheme: mid G represents G4-G6, late G represents G6-G8, F/G represents F8-G2, and so on. A question mark placed next to a classification indicates that the uncertainty in spectral type is greater, so mid G? means $\mathrm{G} 3-\mathrm{G} 7$ and $\mathrm{F} / \mathrm{G}$ ? means $\mathrm{F} 7-\mathrm{G} 3$. A star classified as G? means $\mathrm{G} 0-\mathrm{G} 9$ (and equivalently for other types).

\subsection{Cataclysmic Variables}

The full list of matches between our X-ray and optical source lists having $\mathrm{H} \alpha-R<-0.3, V<23$, and an optical signal-tonoise ratio $>1.4$ is given in Table $6 .{ }^{8}$ We restrict $\mathrm{H} \alpha-R$ based on the work of Szkody et al. (2004), who find that only $17 \%$ of their sample of CVs from the Sloan Survey have an $\mathrm{H} \alpha$ equivalent width below $28 \AA$ in emission (i.e., have $\mathrm{H} \alpha-R>-0.3$ ). We can use spectra to rule out four of these as $\mathrm{dMe}$, normal, or T Tauri stars. The objects ChOPS J174559.18-290418.9 in field SgrA $\star$ and ChOPS J165335.32-393715.9 in field J1655 had no optical spectra taken in any ChaMPlane observing run and so remain uncertain $\mathrm{CV}$ candidates.

Qualitative analysis of the remaining spectra reveals two clear CV candidates in the GalCA field from the LDSS2 spectral sample (see Table 5, sources ChOPS J174638.02-285326.2 and J174656.89-285233.9). Hereafter we refer to these as CV-A and CV-C, respectively (see Figs. $6 a$ and $6 c$ ). Another source, ChOPS J174607.52-285951.3 (hereafter CV-B), was imaged with LDSS2 in the $\mathrm{Sgr} A \star$ field, but the spectrum suffered from being on the edge

\footnotetext{
${ }^{8} \mathrm{CV}-\mathrm{C}$ is included for its broad $\mathrm{H} \alpha$ emission line.
}
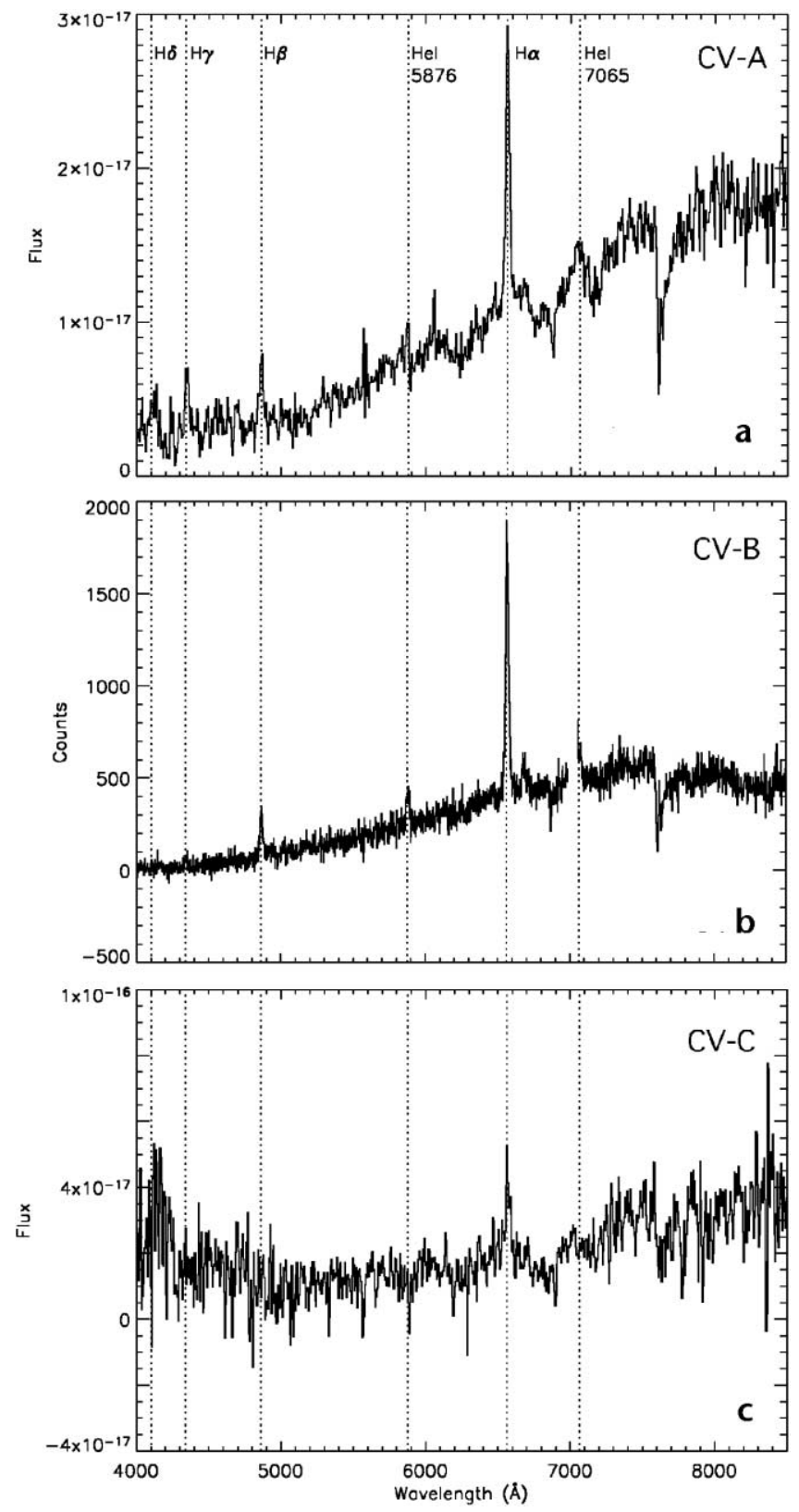

FIG. 6.-Three CVs discovered in the five Chandra fields in this paper. The LDSS2 spectra for CV-A and CV-C have $y$-axis units ergs $\mathrm{s}^{-1} \mathrm{~cm}^{-2} \AA$. The IMACS spectrum for CV-B was not flux-calibrated and has $y$-axis units in raw counts. The region $6990-7500 \AA$ has been removed from CV-B, as it covers a CCD chip gap.

of the slit and was not possible to extract. It was later reobserved by the IMACS instrument at Magellan-its IMACS spectrum is shown in Figure $6 b$. All three spectra show broad $\mathrm{H} \alpha$ in emission. $\mathrm{CV}-\mathrm{A}$ and $\mathrm{CV}-\mathrm{B}$ also show emission lines of $\mathrm{He}$.

CV-B was previously detected by ROSAT Position Sensitive Proportional Counter (PSPC) observations of the GC regions (Sidoli et al. 2001), as their source 65, but without further identification possible at the time. It was found to have a count rate of $1.9 \pm 0.3$ counts $\mathrm{ks}^{-1}$ in the ROSAT band $0.1-2.4 \mathrm{keV}$. Using the online PIMMS tool with an assumed thermal bremsstrahlung spectrum with $k T=7.3 \mathrm{keV}$ and $N_{\mathrm{H}}=5 \times 10^{21} \mathrm{~cm}^{-2}$ from our QCCD analysis (Fig. 4), this converts to a Chandra hard-band $(2-8 \mathrm{keV})$ flux of $1.0 \pm 0.2 \times 10^{-13} \mathrm{ergs} \mathrm{s}^{-1} \mathrm{~cm}^{-2}$. From our Chandra data we estimate $0.64 \pm 0.15 \times 10^{-13} \mathrm{ergs} \mathrm{s}^{-1} \mathrm{~cm}^{-2}$, possibly indicative of some slight variability. 
TABLE 7

CVs with LDSS2 SPECTRA

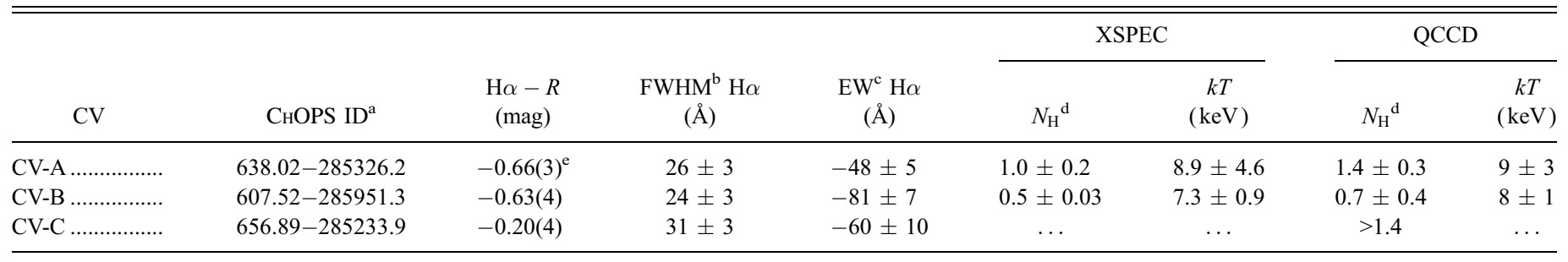

a Abbreviated IDs: prefix ChOPSJ174.

${ }^{b}$ Full width at half-maximum intensity of the $\mathrm{H} \alpha$ line.

${ }^{c}$ Equivalent width of the $\mathrm{H} \alpha$ line.

${ }^{\mathrm{d}} N_{\mathrm{H}}$ in units of $10^{22} \mathrm{~cm}^{-2}$.

e As of 2000. In $2003 \mathrm{H} \alpha-R$ was measured at -0.194 .

We use the QCCD results of Figure 4 to estimate by eye a plausible initial spectral model (CV-A and CV-B were detected in ObsID 945 with $\sim 300$ counts; CV-C has only $\sim 26$ counts). If we assume that a bremsstrahlung spectrum is representative of their X-ray emission, we can thus estimate their spectral properties (see Table 7). We present XSPEC ${ }^{9}$ fits for the two X-ray-bright CVs (CV-A and CV-B) in Figure 7. We use a bremsstrahlung emission plus photon absorption model for the fit to each spectrum. CV-C has too few counts to provide adequate signal-tonoise ratio for spectral fitting. The results we derive are shown in Table 7. For CV-A and CV-B, the quantile-derived parameters are in good agreement with the estimates from the XSPEC fits. For CV-C, we can only place weak constraints on $N_{\mathrm{H}}$ and $k T$, but the error bars are consistent with a typical $\mathrm{CV}$ spectrum of bremsstrahlung at $\sim 2-8 \mathrm{keV}$. This suggests that these $\mathrm{CVs}$ are all dwarf novae (DN) - typically characterized by hot $\left(\sim 10^{8} \mathrm{~K}\right)$ hard X-ray spectra in quiescence (Warner 1995). We note, however, that the absolute magnitudes $M_{V}$ for $\mathrm{CV}-\mathrm{A}$ and $\mathrm{CV}-\mathrm{C}$ are on the bright side for quiescent $\mathrm{DN}$ ( 3 and $<3.99$ respectively).

The final tally of likely CVs is thus one (possibly two) in SgrA $\star$, two in GalCA, and possibly one in the J1655 field. This latter object lies outside the main ACIS-S chip (S3) and its neighboring S4 chip - the large error circle size in which it is found means that even when looking exclusively at objects with $\mathrm{H} \alpha-$ $R<-0.3$ in the field, the probability it is a random match is high $(>20 \%)$. We consider this a low-probability CV candidate.

\subsection{Stellar X-Ray Sources}

In order to compare our sample of stellar X-ray sources with other surveys we construct their luminosity functions and assemble an X-ray-to-optical flux ratio histogram.

Since our survey is flux limited, we correct for incompleteness with the $1 / V_{\max }$ method of Schmidt (1968). In this method, each source contributes 1 over the maximum volume in which it could have been detected in our survey to its bin in the cumulative luminosity histogram. This maximum volume is calculated in the following way: given one ACIS pixel in a Chandra observation, with a known limiting $(3 \sigma)$ count rate for detection (and thus limiting flux, given a spectral model) we can calculate a maximum distance $d_{\max }$, that an object of known luminosity could be placed and still be detected. This defines a pyramidal volume given by the size of the pixel on the sky. To calculate the full volume, we simply repeat this process across the whole field of view, using the $3 \sigma$ count rate limit at each point and sum the

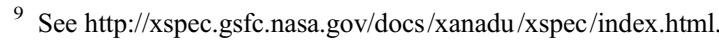

resulting volume elements together. To speed the calculation up we rebin each Chandra image by a factor 80 .

We divide the sources into intermediate (early $F$ to late $G$ ) and late (G/K to M) types, and plot the resulting, $V_{\max }$-corrected luminosity functions in Figure 8 . We use luminosity calculated in the ROSAT band (0.1-2.4 keV) for ease of comparison with past surveys, despite the mismatch in bandpass more suitable to our reddened fields. We overplot comparison luminosity functions of
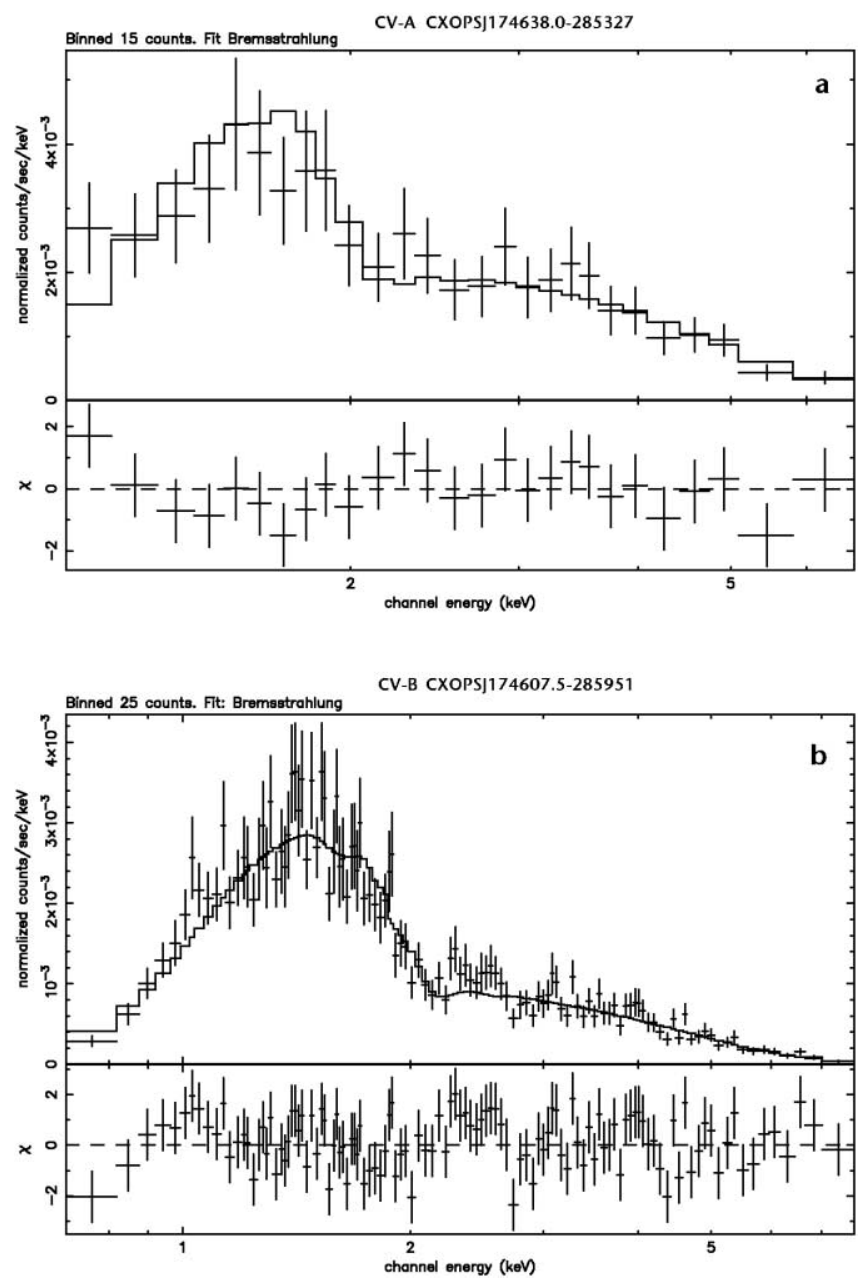

FIG. 7.-XSPEC fits to the X-ray data for CV-A and CV-B detected in this survey. Upper spectrum, CV-A: $438 \pm 22$ net counts ( $B_{\mathrm{X}}$ band); lower spectrum, CV-B: $3539 \pm 63$ net counts. We plot the spectrum plus fit, and in the lower panel in each case the residuals of the spectrum divided by the errors. 

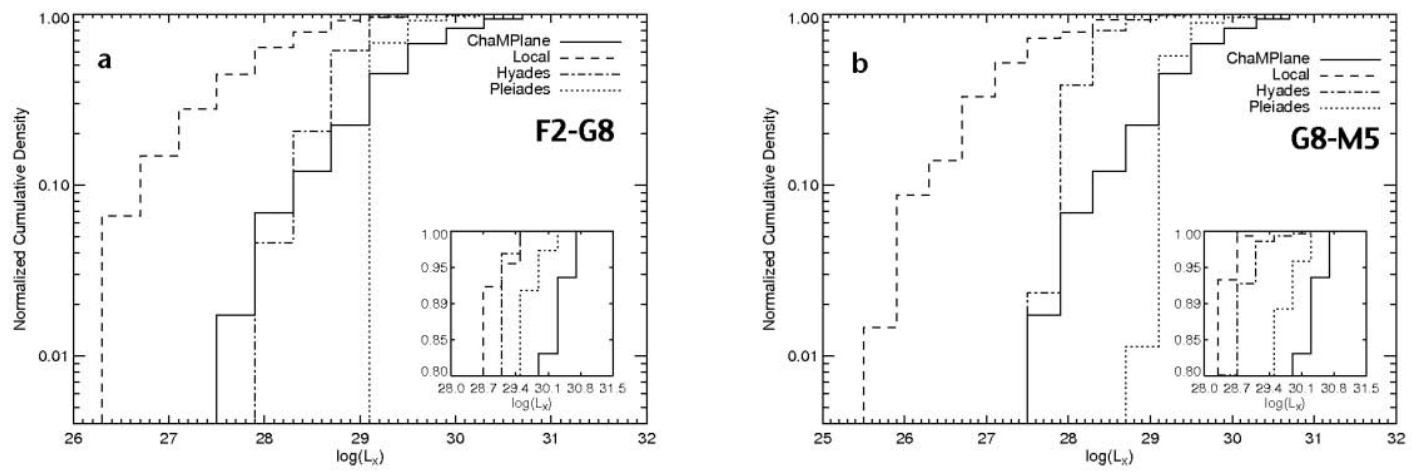

Fig. 8. - X-ray luminosity functions of our stellar coronal sample, $1 / V_{\max }$ corrected. We divide our sample into stars from types F2-G8 (58 stars, left) and G8-M5 (48 stars, right). Inset in each panel is a zoomed-in portion of the top part of the plot. We overplot comparison stellar samples from the a local sample, plus the Hyades and Pleiades.

main-sequence stars (from the volume-limited survey of Schmitt \& Liefke 2004), and young main-sequence stars from the ROSAT surveys of the Hyades (age < 1 Gyr; Stern et al. 1995) and the Pleiades (age $\sim 10^{8}$ yr; Micela et al. 1996). We convert their quoted luminosities into the $0.1-2.4 \mathrm{keV}$ band using the online PIMMS tool. ${ }^{10}$ Since in $\S 3.1$ we concluded that the spectral fit $N_{\mathrm{H}}$ overestimates the true value, for this comparison we generate luminosities with $N_{\mathrm{H}}$ reduced by $0.2 \times 10^{22} \mathrm{~cm}^{-2}$, and consequently distances reduced appropriately given the D01 model.

In Figure 9 we plot a comparative histogram of the X-ray-tooptical flux ratio ( $V$-band flux), i.e., $\log \left(F_{\mathrm{X}} / F_{V}\right)$. Again we utilize the reduced $N_{\mathrm{H}}$ values to generate X-ray rate-to-flux conversions to calculate the X-ray fluxes, and deredden the optical magnitudes. We overplot the samples of Schmitt \& Liefke (2004), Stern et al. (1995), and Micela et al. (1996), scaled so that the total area under each curve is the same for all histograms within a plot.

\subsection{Candidate Low-Mass X-Ray Binary}

A quiescent low-mass X-ray binary (qLMXB) system consisting of a black hole or neutron star and a main-sequence star will most likely show strong $\mathrm{H} \alpha$ in emission in its optical spectrum.

${ }^{10}$ See http://cxc.harvard.edu/toolkit/pimms.jsp.

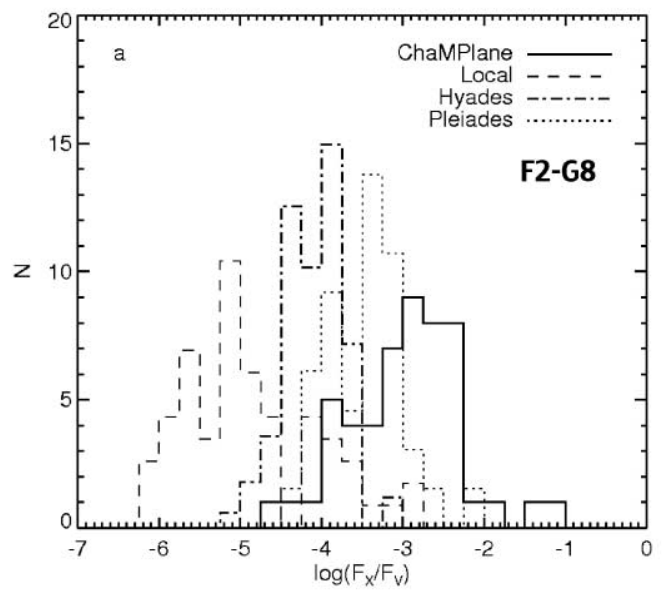

Some CVs and qLMXBs have subgiant companions; these are more likely to be detected in our reddened sample. Since the only objects showing $\mathrm{H} \alpha$ in emission in this sample are either CVs or $\mathrm{T}$ Tauri stars (and identified as such in Table 5), we look to the example of GRO J165540 (Zhang et al. 1994; Harmon et al. 1995; Bailyn et al. 1995), a known qLMXB, soft X-ray transient system, consisting of an F3-6 IV subgiant star secondary and an accreting black hole primary. This system shows $\mathrm{H} \alpha$ in absorption in quiescence: the secondary star is luminous enough to hide the emission line produced by the accretion disk. Our spectral classification is not precise enough to ascertain the luminosity classes of stars in the sample, so to find analogs to this system we search for stars with spectral type earlier than K, with absolute magnitude $M_{V}$ more than $2 \sigma$ higher than that expected for a main-sequence star of that type (see Table 4). We then look for stars with $\log \left(F_{\mathrm{X}} / F_{R}\right)\left(S_{C}\right.$ band $)$ more than $2 \sigma$ greater than that seen in subgiant stars in the survey of Hünsch et al. (1998). In Table 5 we flag the one candidate: ChOPS J165408.14-395636.1 that we find after this search. Figure $10 a$ presents the LDSS2 spectrum. This is only a very tentative classification-it is also possible that this object is an active binary of RS CVn type (Hall 1976). We note that on the basis of its spectrum and X-ray properties in quiescence alone, GRO J1655-40 (see Fig. 10b) could be mistaken for an RS CVn system. Further variability analysis

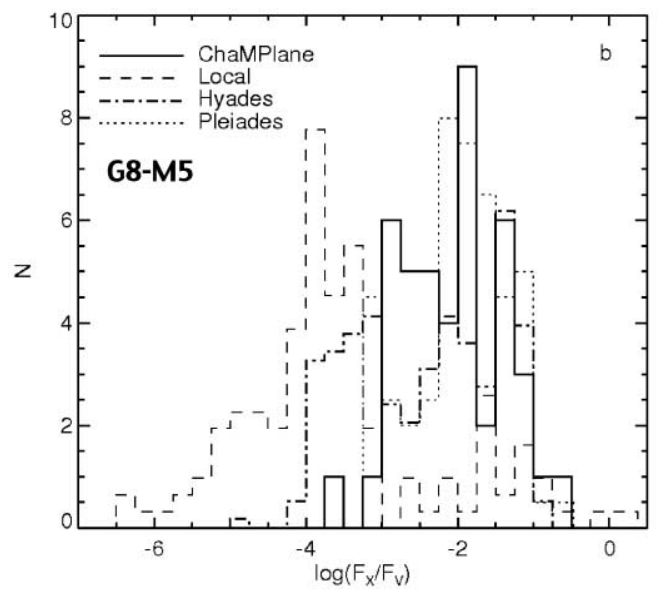

Fig. 9.-Histograms by spectral type grouping (same as Fig. 7) of the X-ray-to-optical flux ratio of our stellar sample, overplotted with data from the studies of Schmitt \& Liefke (2004; dashed) and the Hyades and Pleiades samples of Stern et al. (1995) and Micela et al. (1996). We classify $62 \%-77 \%$ of our M stars as dMe from their $\mathrm{H} \alpha$ emission. 

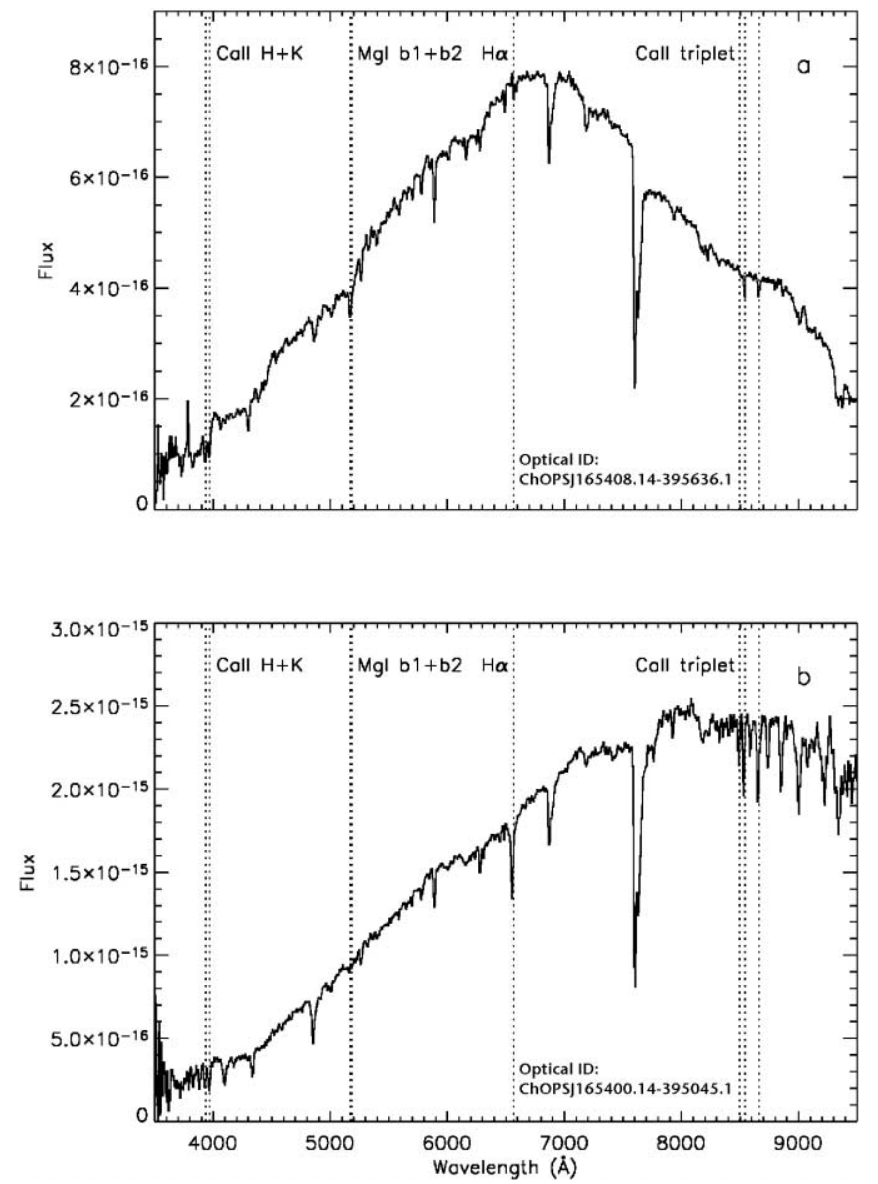

FIG. 10.-Top: qLMXB candidate found in our LDSS2 sample. Bottom: GRO J1655-40 as observed by LDSS2 from our 2002 June observing run. The flux scale has units of ergs s-1 $\mathrm{cm}^{-2} \AA^{-1}$. Important spectral lines are marked on the spectra for reference. The strongest spectral feature at $7600 \AA$ is telluric absorption by the Earth's atmosphere.

and detailed spectral follow-up is necessary to rule out this object as a black hole or neutron star binary system.

\section{DISCUSSION}

\subsection{Stellar Coronal Emission}

We have discovered a large sample of stellar coronal emission sources in our survey fields. Recent studies (e.g., Tagliaferri et al. 1994; Sciortino et al. 1995) have shown that flux-limited X-ray surveys of the Galaxy (within the disk) will naturally sample preferentially from the youngest stellar populations present in the Galactic disk, owing to the known decline of stellar X-ray emission with age (Vaiana et al. 1992). Figures 7 and 8 show that the objects discovered in our survey are somewhat elevated in their X-ray emission, hence one explanation is that they are young. In this picture, the luminosity functions in Figure 8 are possibly explained by a composite of local (old) and younger population (Hyades and Pleiades age) stars. However, the presence of very luminous objects $\left[\log \left(L_{\mathrm{X}}\right)>30\right]$ suggests an additional component, as does the excess of objects at high $\log \left(F_{\mathrm{X}} / F_{V}\right)$ in Figure 9.

The first possibility is that we are detecting an even younger population, in other words a component of pre-main-sequence (pre-MS) stars. Results from ROSAT and the COUP (Getman et al. 2005) show that $T$ Tauri stars (weak-lined and classical) can have X-ray luminosities in excess of $10^{30} \mathrm{ergs} \mathrm{s}^{-1}$, and in some cases greater than $10^{31} \mathrm{ergs} \mathrm{s}^{-1}$. Since our survey covers fields at

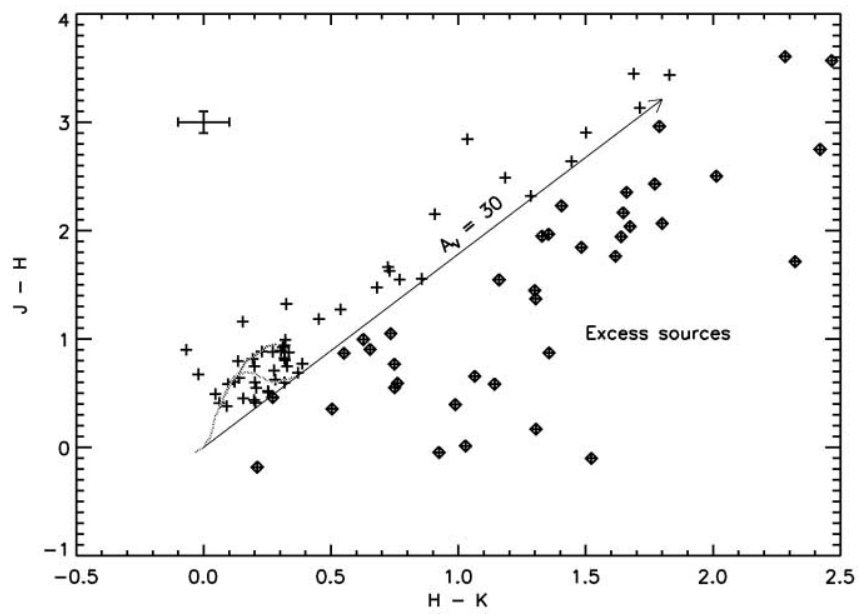

FIg. 11. $-J-H$ vs. $H-K_{S}$ plot of 2 MASS sources matched with ChaMPlane sources in this paper. Overlaid is the locus of dwarf and giant stars (giant stars are redder) from Bessell \& Brett (1988). The arrow represents an extinction of $A_{V}=30$ using the extinction relation of Nishiyama et al. (2006). Sources marked with diamond points are candidate excess sources/young stars. A typical error bar is shown in the upper left.

low Galactic latitude $\left(|b|<3^{\circ}\right)$, and since the scale height of stars also increases with age (Wielen 1977), this is a likely source of X-ray-active objects. To further test the likely pre-MS content of our survey, we examined the near-infrared (near-IR) colors of ChaMPlane objects in this paper (using online data from the 2MASS survey; Skrutskie et al. 2006). In Figure 11 we present a plot of $J-H$ versus $H-K_{S}$ colors obtained in this way. We found 92 2MASS matches to our 136 object source list. Overlaid on the plot are the locus of main-sequence stars and giants from Bessell \& Brett (1988) and a reddening vector created using the near-IR extinction relation of Nishiyama et al. (2006). Objects below the line are candidate infrared excess (T Tauri) young stars (following the work of Lada \& Adams 1992) - a total of between 36 and 56 , or $39 \%-61 \%$ of sources (the range is due to the 2MASS photometric uncertainty). It is also possible for T Tauri stars to be found above the line - this component is not possible to establish without high-resolution spectroscopy to measure $\mathrm{Li}$ $6708 \AA$ absorption equivalent widths.

The other alternative explanation for the X-ray-active objects are active binaries (ABs) of either RS CVn or BY Dra (Bopp \& Fekel 1977) type, or CVs. The studies of Dempsey et al. (1993, 1997) showed that $\log \left(L_{X}\right)$ can range up to 32 for RS CVn and BY Dra systems, with $\log \left(F_{\mathrm{X}} / F_{V}\right)$ up to -1.0 . CVs also typically have $\log \left(F_{\mathrm{X}} / F_{V}\right) \sim-3$ to +1 (Verbunt et al. 1997); however, the lack of broad $\mathrm{H} \alpha$ argues against $\mathrm{CV}$ s unless they have subgiant companions. To estimate the likely contribution of $\mathrm{ABs}$ to our sample, we use a method similar to that used by Grindlay et al. (2005). We define a maximum distance at which an $A B$ would have been identified given the optical and X-ray detection limits of our survey. Given some model for the distribution of $\mathrm{ABs}$ in the Galaxy, we can predict the number we expect to detect in the corresponding volume.

The detectability of a given $\mathrm{AB}$ in our Chandra observations is determined by the detection limit of the observation, the hydrogen column intervening between the telescope and the object, its luminosity, and the spectral model assumed for its emission.

To quantify the Chandra sensitivity in the calculation, we extract the count rate limit for each observation across the field of view. For simplicity we use an average value across the detector for each field in the $B_{\mathrm{X}}$ band. We derive the hydrogen column density $N_{\mathrm{H}}$ encountered as a function of distance using the D01 
calculation of $A_{V}$ in the direction of the aim point of each field. As a simple approximation, we assume that ABs are distributed in the Galaxy with some exponential scale height $h$ in the $z$-direction: $n_{\mathrm{AB}} \propto \exp ^{-d(\sin |b|) / h}$, with $n_{\mathrm{AB}}$ the $\mathrm{AB}$ space density and $b$ the Galactic latitude. This is probably a reasonable assumption for the regions surveyed in this paper (provided we are only considering the distribution within $\sim 3 \mathrm{kpc}$ of the solar neighborhood). Following Grindlay et al. (2005) we consequently utilize the formalism of Tinney et al. (1993) in constructing an effective detection volume $V_{\text {eff }}$, as defined by $d_{\max }$ :

$$
V_{\mathrm{eff}}=\Omega(h / \sin |b|)^{3}\left[2-\left(\chi^{2}+2 \chi+2\right) \exp (-\chi)\right]
$$

where $\chi=d_{\max }(\sin |b|) / h$ and $\Omega$ is the solid angle subtended by the ACIS field of view. The $V_{\text {eff }}$ corrects the geometric volume in which we search for the nonuniformity of the AB space density. The $d_{\max }$ value is the limiting distance at which an $\mathrm{AB}$ at a luminosity of $10^{32.0} \mathrm{ergs} \mathrm{s}^{-1}$ (the maximum value for $\mathrm{ABs}$ found by ROSAT; Dempsey et al. 1993) could be detected in the Chandra observation considered. For the ACIS-S observation J1655, we use the full $8^{\prime} \times 8^{\prime}$ field of view to calculate $\Omega$. The GalCA pointing overlaps the SgrA $\star$ field of view, so this field only adds three-quarters of the full ACIS-I solid angle to the area of sky surveyed. The number of $\mathrm{ABs}$ we might expect to be present in such a volume is then $N_{\mathrm{AB}}=n_{\mathrm{AB}} V_{\text {eff }}$.

Following the conclusions of Sciortino et al. (1995), we adopt an $\mathrm{AB}$ scale height of $h=250 \mathrm{pc}$, and a local space density $n_{\mathrm{AB}}=3.7 \times 10^{-5} \mathrm{pc}^{-3}$ (Favata et al. 1995). For the X-ray spectral model, we adopt a MEKAL $k T=1.0 \mathrm{keV}$ single-temperature model. To model the detection rate of $\mathrm{ABs}$, we populate each volume uniformly with a randomly distributed sample of $10^{4} \mathrm{ABs}$ from $0 \mathrm{pc}$ up to $d_{\max }$ as determined for each field. Each is assigned an X-ray luminosity and absolute visual magnitude, sampling randomly from the data of Dempsey et al. $(1993,1997)$ using only objects they classify as RS CVn or BY Dra type. This enables us to determine if an object is luminous enough in X-rays and apparent visual magnitude to be detected in our survey (using an assumed detection limit of $V=21$ for an object to be spectrally classified). Combining the detection rate derived with the predicted number of $A B$ s in each volume, we predict between 13 and $14 \mathrm{ABs}$ found in our survey. Based on absolute visual magnitude and high $\log \left(F_{\mathrm{X}} / F_{\text {opt }}\right)$ ratio, ${ }^{11}$ we flag our best $\mathrm{AB}$ candidates in Table 5.

Our stellar coronal source sample appears likely to be a mix of both local, young- and pre-MS stars, and a component of coronally active binaries: RS CVn and BY Dra type. To specifically compare the stars of these types in our sample with those found by $R O S A T$ would require significantly improved spectral, variability, and orbital analysis to more precisely classify our objects and tease out the contributions of age, metallicity, and binarity that might also be contributing to the observed differences in the luminosity and X-ray-to-optical flux ratio.

\subsection{Constraints on the Galactic CV Density}

A main aim of the ChaMPlane survey is to investigate what constraints we can place on the local CV space density. We follow a method similar to that described in $\S 5.1$.

11 RS CVn: $M_{V}$ more than $2 \sigma$ less than that expected for a main-sequence star of that spectral type and $\log \left(F_{\mathrm{X}} / F_{R}\right)>-5.0$. BY Dra: $M_{V}$ consistent with main sequence, $\log \left(F_{\mathrm{X}} / F_{R}\right)$ more than $2 \sigma$ greater than that expected for its spectral type (Hünsch et al. 1998).
For the Chandra sensitivity in the calculation, we use a single value for the Chandra $H_{C}$ band, averaging over a $5^{\prime}$ radius circle centered on the aim point. For the X-ray spectral model, we adopt a $k T=8 \mathrm{keV}$ bremsstrahlung emission spectrum as "typical" for dwarf nova CVs (Warner 1995). In this case, $d_{\max }$ is the limiting distance at which a CV at a luminosity of $10^{32.5} \mathrm{ergs} \mathrm{s}^{-1}$ (the maximum value for CVs found by ROSAT; Verbunt et al. 1997) could be detected in the Chandra observation considered.

We adopt a scale height of $h=200 \mathrm{pc}$, and, following the conclusions of Grindlay et al. (2005) among others, we adopt a local space density $n_{\mathrm{CV}}=1 \times 10^{-5} \mathrm{pc}^{-3}$. We populate each volume uniformly with a randomly distributed sample of $10^{5} \mathrm{CVs}$ from $0 \mathrm{pc}$ up to $d_{\max }$ as determined for each field. We assign each fake $\mathrm{CV}$ an X-ray luminosity (in the $H_{C}$ band) and X-ray-tooptical flux ratio $\log \left(F_{\mathrm{X}} / F_{V}\right)$ : we sample $L_{\mathrm{X}} \mathrm{X}$-ray data as collected by Grindlay et al. (2005), originally presented in Hertz et al. (1990) and the ROSAT survey (see Verbunt et al. 1997; Schwope et al. 2002), to construct distributions in $L_{X}$ and $\log \left(F_{X} / F_{V}\right)$ from which we randomly sample. We assume that these two parameters are uncorrelated (a simple scatter plot shows this to be the case for the $49 \mathrm{CVs}$ in the ROSAT sample). For a bremsstrahlung X-ray spectral model at $8 \mathrm{keV}$, the luminosity in the ROSAT band $0.1-2.4 \mathrm{keV}$ and our $H_{C}$ band is approximately the same. We then derive an apparent $V$ magnitude and observed $\mathrm{X}$-ray flux. We set our photometric detection limit at a $V$ magnitude of 23 - the faintest that we could have detected a $\mathrm{CV}$ via its $\mathrm{H} \alpha-R$ color. The number of CVs expected to be present in each field is given in Table 8 as " $\mathrm{CV}_{32.5}$ " (col. [5]). The X-ray detection and optical identification percentages of these objects are given in columns (6) and (7). The resulting number of predicted CV detections is given as " $\mathrm{ID}_{32.5}$." Since the optical detection limit is reached at only $\sim 2 \mathrm{kpc}$, the optical percentage quoted below is essentially equal to the combined "X-ray-and-optical" detection percentage.

It is apparent that there is an excess of CV candidates over the number predicted by our simulation. In SgrA $\star$ and GalCA fields considered in isolation there are a factor $\sim 10-70$ too many. However, summing over all fields we find between three and five detected, with $0.95 \pm 0.08$ predicted. A simple $\chi^{2}$ table shows that this result is statistically significant at $\sim 92 \%$ confidence. What factors are contributing to the discrepant estimation of the $\mathrm{CV}$ detection rate?

We have assumed that a single relationship between $A_{V}$ and distance is applicable over each $16^{\prime} \times 16^{\prime}$ Chandra field of view, yet extremes in the level of extinction are observed directly in infrared images of the SgrA $\star$ and GalCA fields (see, e.g., Laycock et al. 2005). The D01 model overlooks this small-scale variability across each field. If there were some covering factor of higher column density gas and dust across each field or regions of significantly lower extinction, we would expect to alter the number of predicted CVs detected. However, a factor of $\sim 15$ decrease in the amount of extinction as a function of distance is necessary to produce the factor $\sim 60$ increase in the overall prediction for $\mathrm{CV}$ detections in GalCA, and at least a factor $\sim 5$ reduction is required to solve the discrepancy for SgrA $\star$. Although there is some great uncertainty in the dust model toward the Galactic center, this appears unlikely. Improvements to the dust model are vital to understand if this effect is more significant.

We included no radial component in our model CV space distribution, yet it is likely that there is some increase in the space density of CVs as we approach the Galactic center (see, e.g., Rogel et al. 2008). Thus, we are underestimating the true number of CVs present in each volume, before we apply our detection criteria. This may only be a minor correction, since our optical 
TABLE 8

CV Detection Constraints for the Fine Chandra ObsiDs

\begin{tabular}{|c|c|c|c|c|c|c|c|c|}
\hline $\begin{array}{l}\text { ObsID } \\
\text { (1) }\end{array}$ & $\begin{array}{l}\text { Field } \\
\text { (2) }\end{array}$ & $\begin{array}{c}\mathrm{CR} \mathrm{Limit}^{\mathrm{a}} \\
\left(\mathrm{ks}^{-1}\right) \\
(3)\end{array}$ & $\begin{array}{c}d \max _{32.5} \mathrm{~b} \\
(\mathrm{kpc}) \\
(4)\end{array}$ & $\begin{array}{c}\mathrm{CV}_{32.5^{\mathrm{c}}} \\
\text { (5) }\end{array}$ & $\begin{array}{c}\text { X-Detect }{ }^{\mathrm{d}} \\
(\%) \\
(6)\end{array}$ & $\begin{array}{c}\text { XO-Detect } \\
\text { (\%) } \\
(7)\end{array}$ & $\begin{array}{c}\mathrm{ID}_{32.5}{ }^{\mathrm{f}} \\
(8)\end{array}$ & $\begin{array}{l}\text { Found } \\
\text { (9) }\end{array}$ \\
\hline 99.................... & $\mathrm{J} 1655$ & 0.258 & 29.3 & 21.2 & $12.3 \pm 0.2$ & $2.28 \pm 0.03$ & 0.48 & $0(1)^{g}$ \\
\hline 737......................... & G347b & 0.305 & 15.75 & 37.3 & $4.1 \pm 0.2$ & $0.65 \pm 0.03$ & 0.24 & 0 \\
\hline $944 \ldots \ldots$ & SgrB2 & 0.126 & 14.4 & 65.8 & $3.4 \pm 0.2$ & $0.05 \pm 0.01$ & 0.03 & 0 \\
\hline 945......................... & GalCA & 0.305 & 11.3 & 22.7 & $3.0 \pm 0.2$ & $0.13 \pm 0.02$ & 0.03 & 2 \\
\hline $53392 \ldots \ldots \ldots \ldots \ldots \ldots$ & SgrA $\star$ & 0.061 & 39.5 & 1232.0 & $1.7 \pm 0.02$ & $0.014 \pm 0.002$ & 0.17 & $1(1)^{\mathrm{g}}$ \\
\hline Total ...................... & $\ldots$ & $\ldots$ & $\ldots$ & 1378.8 & $\ldots$ & $\ldots$ & 0.95 & $3(5)$ \\
\hline
\end{tabular}

${ }^{\text {a }}$ The average count rate limit for this observation within $5^{\prime}$ of the Chandra aim point.

b The maximum distance we can detect a CV at $\log \left(L_{\mathrm{X}}\right)=32.5$.

c The number of CVs in the effective volume defined by the Tinney formula for this sky position and $d$ max.

${ }^{\mathrm{d}}$ The expected percentage of those CVs detected in X-rays.

e The expected percentage of those CVs also detected optically.

${ }^{\mathrm{f}}$ The resultingnumber of CVs we expect to identify in this field.

g This field includes one spectroscopically unconfirmed CV; see Table 6.

detection and spectroscopic identification limit restricts us to looking in the nearest $\sim 2 \mathrm{kpc}$ to the Sun. Some additional work on improving how we model where the CVs are in our volume, and how many we expect to be in this volume, is important to establish by how much we are underestimating $N_{\mathrm{CV}}$. Such a modification would appear to be most necessary for the two fields closest to the Galactic center: SgrA $\star$ and GalCA.

We restricted the detection solid angle to the inner $5^{\prime}$ of the Chandra field of view. Including the outer parts of the detector (or for ACIS-S including the other S-chips) increases the detection area by a factor $\sim 3$. However, the X-ray count rate limit beyond $5^{\prime}$ is lower (on average by a factor $\sim 2$ ) than at the aim point, which, although not affecting optical detectability, means the increase in predicted CV numbers would be small in comparison with other effects discussed above.

J. Patterson (2004, private communication) recommends a smaller scale height $h=150 \mathrm{pc}$ on the basis of local CV surveys. Implementing this affects only field J1655 significantly and would reduce the predicted number of CVs by $\sim 20 \%$ - further in line with our lack of CVs detected in this field.

At face value, our results suggest a higher than local value of the $\mathrm{CV}$ density, $n_{\mathrm{CV}}$, although alternatively the excess of objects detected may simply represent fluctuations over a mean background rate. The parameter $n_{\mathrm{CV}}$ directly influences the predicted number of CVs in any given field. Our sample is too small to be used to argue strongly for a change; however, the significance of the difference between the observed number of CVs and our predicted number certainly suggests an increase (by a factor $\sim 3-5$ ) is justified. Two recent studies find different values for $n_{\mathrm{CV}}$. Rogel et al. (2008) construct a Galaxy source distribution model to predict $\mathrm{CV}$ detections found in ChaMPlane Galactic anticenter fields, and find a value of $10^{-5} \mathrm{pc}^{-3}$ provides the best fit to their results. However, Ak et al. (2008) use a sample of 459 local solar neighborhood CVs to derive a value of $n_{\mathrm{CV}}$ a factor $\sim 3$ higher than this, which appears to validate our own findings. Thus, it may indeed be that the value of $n_{\mathrm{CV}}$ is somewhat higher than our adopted value.

\subsection{Resolving the Galactic Ridge X-Ray Emission}

The extended Galactic ridge X-ray emission (GRXE; see, e.g., Worrall et al. 1982) observed throughout the Galactic plane is the subject of disagreement over whether deeper, higher resolution observations in the X-ray band will eventually completely resolve all observed Galactic emission at this wavelength, or whether in fact there is a truly diffuse emitting plasma confined to the Galactic plane. Ebisawa et al. (2005) carried out deep, $\sim 100 \mathrm{ks}$ X-ray observations of two fields in the Galactic plane with Chandra and estimated that point sources contribute at most $10 \%$ of the total X-ray flux they observe in this region. They conclude that no faint, unobserved point-source population could simultaneously match their observed $\log N-\log S$ (number vs. source flux) plots of point sources and the total GRXE flux, and hence there is a truly diffuse component to the GRXE. On the other hand, Revnivtsev et al. (2006) show that, since the morphology of the GRXE very closely matches that of the Galactic nearinfrared surface brightness, it must trace the stellar mass distribution of the Galaxy. They calculate the X-ray luminosity per unit stellar mass that this conclusion requires in order to match the observed flux of the GRXE. Together with the X-ray luminosity function of Sazonov et al. (2006) they show that the X-ray emissivity per unit mass of the local solar neighborhood X-ray source population extended to the whole Galaxy can easily account for the GRXE.

The probable types of the counterparts to our X-ray source population are active binaries ( $\sim 10 \%$ of the sample), CVs $(\sim 2 \%)$, and YSOs (perhaps between $39 \%$ and $61 \%$ ), with the remainder coronally emitting stars, either young main-sequence or mainsequence objects. Broadly and qualitatively this result agrees with the make-up of the solar neighborhood population as determined by Sazonov et al. (2006). A more detailed comparison with their luminosity function is limited by two factors: (1) the fact that our survey is not complete in either magnitude/source flux space or volume space, and (2) the lack of detailed source classification and hence a determination of the X-ray emissivity per stellar mass of our sources. However, our results suggest a significant contribution to X-ray-emitting sources close to the Galactic plane comes from pre-main-sequence and young main-sequence stars, which can have relatively high X-ray luminosities $\left(L_{\mathrm{X}} \sim 10^{31} \mathrm{ergs} \mathrm{s}^{-1}\right)$.

In order to make progress to address the conflict over the origin of the GRXE requires one or both of deep X-ray observations ( $\sim 1 \mathrm{Ms}$; Revnivtsev et al. 2006) or accurate classification (via optical or infrared spectroscopy or X-ray spectral analysis) of the faint, hard X-ray source population present in many Chandra X-ray studies of the Galactic plane (e.g., Muno et al. 2004). Although our Galactic center pointing approaches the first requirement, it still falls somewhat short and is hindered by the large extinction toward this field $\left[\log \left(N_{\mathrm{H}}\right) \approx 23.0 \mathrm{~cm}^{-2}\right]$. Since we use optical spectroscopy to classify targets, we are necessarily 
limited to relatively bright $(R \lesssim 21)$, nearby objects and as a result preferentially observe soft X-ray-emitting, more unabsorbed sources. A forthcoming ChaMPlane paper (J. Hong et al. 2008, in preparation) will utilize very deep ( $\sim 1 \mathrm{Ms})$ Chandra observations of the Galactic center together with our quantile analysis technique to classify faint point sources to directly consider the origin of the GRXE.

\section{CONCLUSION}

We have carried out optical and X-ray spectral analysis on a sample of X-ray-detected optical sources in the Galactic plane, using a combination of optical spectral fitting and quantile X-ray analysis to obtain the extinction $E(B-V)$ and hence $A_{V}$ and hydrogen column density $N_{\mathrm{H}}$ toward each object. We combine these estimates with the work of D01, who present a threedimensional dust model of the Galaxy in order to derive $A_{V}$ as a function of distance in any direction, and thus further derive a distance to each object.

We present the discovery of a population of stellar coronal emission sources, detected by Chandra in five fields toward the Galactic bulge. These are likely a mix of young stars, of roughly Hyades and Pleiades age, as well as some pre-MS stars, and a component of RS CVn or BY Dra type. We find no strong evidence that we have sampled from stars with significantly different properties from local, similarly active stars. We report the properties of the most probable RS CVn- and BY Dra-type candidates from our sample, and identify one possible qLMXB candidate also. We note that this latter object could instead be an RS CVn system. High-resolution optical spectra can make this clear.

We report the discovery of three X-ray-detected CVs in the direction of the Galactic center. All three are consistent with hav- ing an X-ray spectrum consisting of bremsstrahlung at $k T \approx 8 \mathrm{keV}$, and are within $\approx 2 \mathrm{kpc}$ of the Sun. An additional two CVs are indicated by our photometry and X-ray data, and can be tested with optical spectroscopy.

The number of CVs detected in our survey is consistent with a local CV space density of $\sim 10^{-5} \mathrm{pc}^{-3}$, and a scale height $\sim 200 \mathrm{pc}$, but is suggestive of a larger local value or strong radial gradient. However, there is considerable uncertainty in the model we use to predict extinction as a function of distance and hence derive the number detected in our survey. Although the numerical uncertainty in the model appears to be a factor of $\sim 3$ (see Fig. 5), cf. the required factor $\sim 5-15$ to rectify the discrepancies seen in the GalCA and SgrA fields, it is possible that true variations in the distribution of dust in the Galaxy might be able to explain this. Further work on better modeling the Galactic dust distribution and $\mathrm{CV}$ content of our fields is desirable to improve our constraints.

The authors would like to thank John Silverman for collecting the LDSS2 spectral data during the 2001 run; J. E. G. collected the LDSS2 spectra in the 2002 run. We also thank the two anonymous referees and Eric Feigelson, whose suggestions considerably improved the manuscript. This research has made use of the SIMBAD database, operated at CDS, Strasbourg, France, and the NASA Astrophysics Data System. This work is supported in part by NASA/Chandra grants AR1-2001X, AR2-3002A, AR3-4002A, AR4-5003A, and AR6-7010X, NSF grant AST 00-98683, and the Chandra X-ray Center. We thank NOAO for its support via the Long Term Survey program.
Ak, T., Bilir, S., Ak, S., \& Eker, Z. 2008, NewA, 13, 133

Andrillat, Y., Jaschek, C., \& Jaschek, M. 1995, A\&AS, 112, 475

Bailyn, C. D., Orosz, J. A., McClintock, J. E., \& Remillard, R. A. 1995, Nature, 378, 157

Bessell, M. S. 1991, AJ, 101, 662

Bessell, M. S., \& Brett, J. M. 1988, PASP, 100, 1134

Bopp, B. W., \& Fekel, Jr., F. 1977, AJ, 82, 490

Brand, J., \& Blitz, L. 1993, A\&A, 275, 67

Carquillat, M. J., Jaschek, C., Jaschek, M., \& Ginestet, N. 1997, A\&AS, 123,5

Cox, A. N. 2000, Allen's Astrophysical Quantities (4th ed.; New York: Springer)

Dame, T. M., Hartmann, D., \& Thaddeus, P. 2001, ApJ, 547, 792

Dempsey, R. C., Linsky, J. L., Fleming, T. A., \& Schmitt, J. H. M. M. 1993, ApJS, 86, 599

$$
\text { 1997, ApJ, 478, } 358
$$

Dickey, J. M., \& Lockman, F. J. 1990, ARA\&A, 28, 215

Dopita, M. A., \& Sutherland, R. S. 2003, Astrophysics of the Diffuse Universe (Berlin: Springer)

Drimmel, R., Cabrera-Lavers, A., \& López-Corredoira, M. 2003, A\&A, 409, 205 (D03)

Drimmel, R., \& Spergel, D. N. 2001, ApJ, 556, 181 (D01)

Ebisawa, K., et al. 2005, ApJ, 635, 214

Favata, F., Micela, G., \& Sciortino, S. 1995, A\&A, 298, 482

Getman, K. V., et al. 2005, ApJS, 160, 319

Gray, D. F. 1992, The Observation and Analysis of Stellar Photospheres (2nd. ed; Cambridge: Cambridge Univ. Press)

Grindlay, J. E., et al. 2003, Astron. Nachr., 324, 57 2005, ApJ, 635, 920

Hall, D. S. 1976, in IAU Colloq. 29, Multiple Periodic Variable Stars, ed. W. S. Fitch (Dordrecht: Reidel), 287

Harmon, B. A., et al. 1995, Nature, 374, 703

Hertz, P., Bailyn, C. D., Grindlay, J. E., Garcia, M. R., Cohn, H., \& Lugger, P. M. 1990, ApJ, 364, 251

Hong, J., Schlegel, E. M., \& Grindlay, J. E. 2004, ApJ, 614, 508 (H04)

Hong, J., van den Berg, M., Schlegel, E. M., Grindlay, J. E., Koenig, X., Laycock, S., \& Zhao, P. 2005, ApJ, 635, 907

\section{REFERENCES}

Houk, N., Swift, C. M., Murray, C. A., Penston, M. J., \& Binney, J. J. 1997, in Hipparcos_-Venice '97, ed. B. Battrick (ESA SP-402; Noordwijk: ESA), 279 Howarth, I. D. 1983, MNRAS, 203, 301

Hünsch, M., Schmitt, J. H. M. M., \& Voges, W. 1998, A\&AS, 132, 155

Jacoby, G. H., Hunter, D. A., \& Christian, C. A. 1984, ApJS, 56, 257

Johnson, H. L. 1966, ARA\&A, 4, 193

Lada, C. J., \& Adams, F. C. 1992, ApJ, 393, 278

Laycock, S., Grindlay, J., van den Berg, M., Zhao, P., Hong, J., Koenig, X., Schlegel, E. M., \& Persson, S. E. 2005, ApJ, 634, L53

Mewe, R., Gronenschild, E. H. B. M., \& van den Oord, G. H. J. 1985, A\&AS, 62, 197

Micela, G., Sciortino, S., Kashyap, V., Harnden, F. R., Jr., \& Rosner, R. 1996, ApJS, 102, 75

Mikami, T., \& Heck, A. 1982, PASJ, 34, 529

Muno, M. P., et al. 2003, ApJ, 589, 225 2004, ApJ, 613, 326

Nishiyama, S., et al. 2006, ApJ, 638, 839

Predehl, P., \& Schmitt, J. H. M. M. 1995, A\&A, 293, 889

Rogel, A. B., Cohn, H. N., \& Lugger, P. M. 2008, ApJ, 675, 373

Revnivtsev, M., Sazonov, S., Gilfanov, M., Churazov, E., \& Sunyaev, R. 2006, A\&A, 452, 169

Sazonov, S., Revnivtsev, M., Gilfanov, M., Churazov, E., \& Sunyaev, R. 2006, A\&A, 450, 117

Schmidt, M. 1968, ApJ, 151, 393

Schmitt, J. H. M. M., \& Liefke, C. 2004, A\&A, 417, 651

Schwope, A. D., Brunner, H., Buckley, D., Greiner, J., Heyden, K. v. d.,

Neizvestny, S., Potter, S., \& Schwarz, R. 2002, A\&A, 396, 895

Sciortino, S., Favata, F., \& Micela, G. 1995, A\&A, 296, 370

Sidoli, L., Belloni, T., \& Mereghetti, S. 2001, A\&A, 368, 835

Silva, D. R., \& Cornell, M. E. 1992, ApJS, 81, 865

Skrutskie, M. F., et al. 2006, AJ, 131, 1163

Stern, R. A., Schmitt, J. H. M. M., \& Kahabka, P. T. 1995, ApJ, 448, 683

Szkody, P., et al. 2004, AJ, 128, 1882

Tagliaferri, G., Cutipoto, G., Pallavicini, R., Randich, S., \& Pasquini, L. 1994, A\&A, 285, 272

Taylor, A. R., et al. 2003, AJ, 125, 3145 
Tinney, C. G., Reid, I. N., \& Mould, J. R. 1993, ApJ, 414, 254

Torres-Dodgen, A. V., \& Weaver, W. B. 1993, PASP, 105, 693

Vaiana, G. S., Maggio, A., Micela, G., \& Sciortino, S. 1992, Mem. Soc. Astron. Italiana, 63, 545

Verbunt, F., Bunk, W. H., Ritter, H., \& Pfeffermann, E. 1997, A\&A, 327, 602

Warner, B. 1995, Cataclysmic Variable Stars (Cambridge: Cambridge Univ. Press)

Wielen, R. 1977, A\&A, 60, 263
Worrall, D. M., Marshall, F. E., Boldt, E. A., \& Swank, J. H. 1982, ApJ, 255, 111

Zhang, S. N., Wilson, C. A., Harmon, B. A., Fishman, G. J., Wilson, R. B., Paciesas, W. S., Scott, M., \& Rubin, B. C. 1994, IAU Circ., 6046, 1

Zhao, P., Grindlay, J. E., Hong, J. S., Laycock, S., Koenig, X. P., Schlegel, E. M., \& van den Berg, M. 2005, ApJS, 161, 429 